PART VIII

CHOICE OF STANDARD STARS 


\title{
REMARKS ON THE PHOTOMETRIC CRITERIA OF CHOICE OF THE STANDARD STARS
}

\author{
M. GOLAY \\ Observatoire de Genève, Switzerland
}

\begin{abstract}
We propose, in several tables, a number of stars selected to be observed both by photometrists, spectroscopists, astrometrists. Properties of selected stars are discussed.
\end{abstract}

\section{Definition of the 'HR Standard' Star}

In astronomy we are familiar with several kinds of 'standard' stars, for instance: standard stars for spectral classification, those destined to define the scales of magnitudes and colours, stars selected to define an absolute energy distribution, etc. Practical considerations have often determined the choice of standard stars: uniform distribution in the sky, easy identification, necessity to cover a certain interval of magnitudes or colours. We propose here, in several tables, a number of stars selected to be observed by photometrists, spectroscopists and astrometrists. This list is of course not exhaustive. We will henceforth name the stars of our list 'HR standard' stars. Indeed, the object is to gather a sufficient amount of observations on each one of these stars to allow the precise fitting of stellar models liable to give us the absolute magnitude $M_{v}$, the gravity $\log g$, the effective temperature $T_{\text {eff }}$, the chemical composition $\chi$. It would be ideal to dispose of these informations for typical stars distributed throughout a three-dimensional HR diagram $\left(M_{v}, T_{\text {eff }}, \chi\right)$. The three physical parameters $M_{v}, T_{\text {eff }}, \chi$ are generally obtained by means of indirect methods, very often photometrically. One must thus bear in mind that the photometric quantities such as magnitudes, indices, combinations of indices, depend in no negligible manner on multiplicity, rotational velocity of the star observed, on the quantity of interstellar matter and on the extinction law. Consequently, care must be given to avoid the use as 'HR standard' of stars having particularities liable to introduce errors in the photometric quantities.

\section{Photometric Effects of Binarity, Rotation, Gravity and Chemical Composition}

In the following diagrams, Figure 1a, b, Figure 2, Figure 3, Figure 4a, b, c, Figure $5 \mathrm{a}, \mathrm{b}$, we attempt to illustrate the deviations in colour to be expected for two stars slightly different in spectral class, or in luminosity class, or in chemical composition, or in rotational velocity, or due to the fact that one of them can be a binary. The conclusion derived from an inspection of these diagrams is that a difference in colour (between $3500 \AA$ and $6500 \AA$ ) of \pm 0.02 can be caused by:

a difference of 0.05 in spectral class;

a difference of $200^{\circ}$ to $300^{\circ}$ in $T_{\text {eff }}$; 

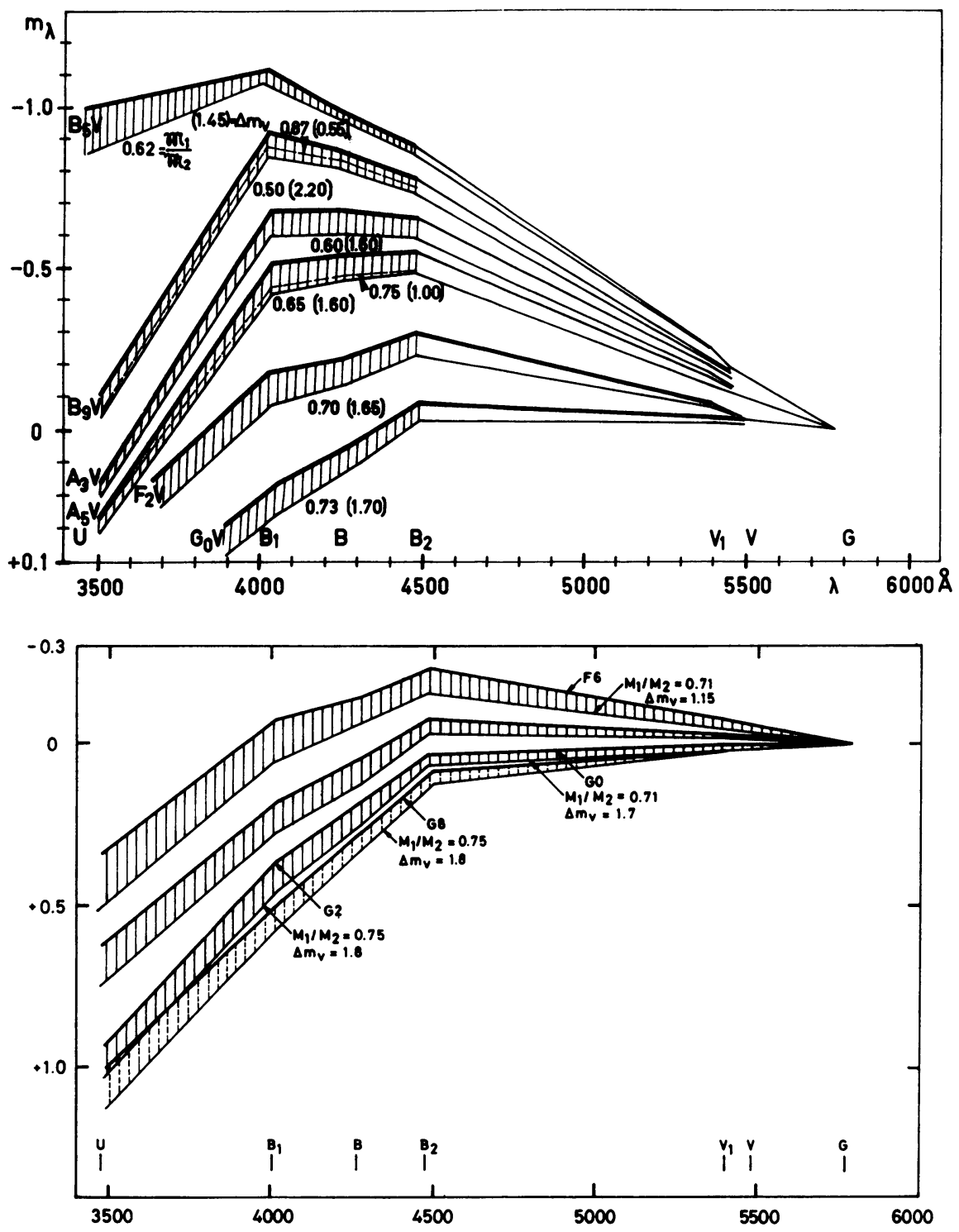

Fig. 1a, b. Effect of binarity for some spectral types. The thick solid line refers to a unit mass ratio. The thin solid line is for a mass ratio giving a pseudo-continuum of greatest deviation from the unit mass ratio.

a difference of 0.3 in $\log g$;

a difference of 0.1 in $[\mathrm{Fe} / \mathrm{H}]$;

a difference of $150 \mathrm{~km} \mathrm{~s}^{-1}$ in rotational velocity;

a companion weaker by $5 \cdot \mathrm{mag}$.

These figures are only orders of magnitude, and can vary considerably individually according to the type of star (cold star, hot star, dwarf or supergiant). 


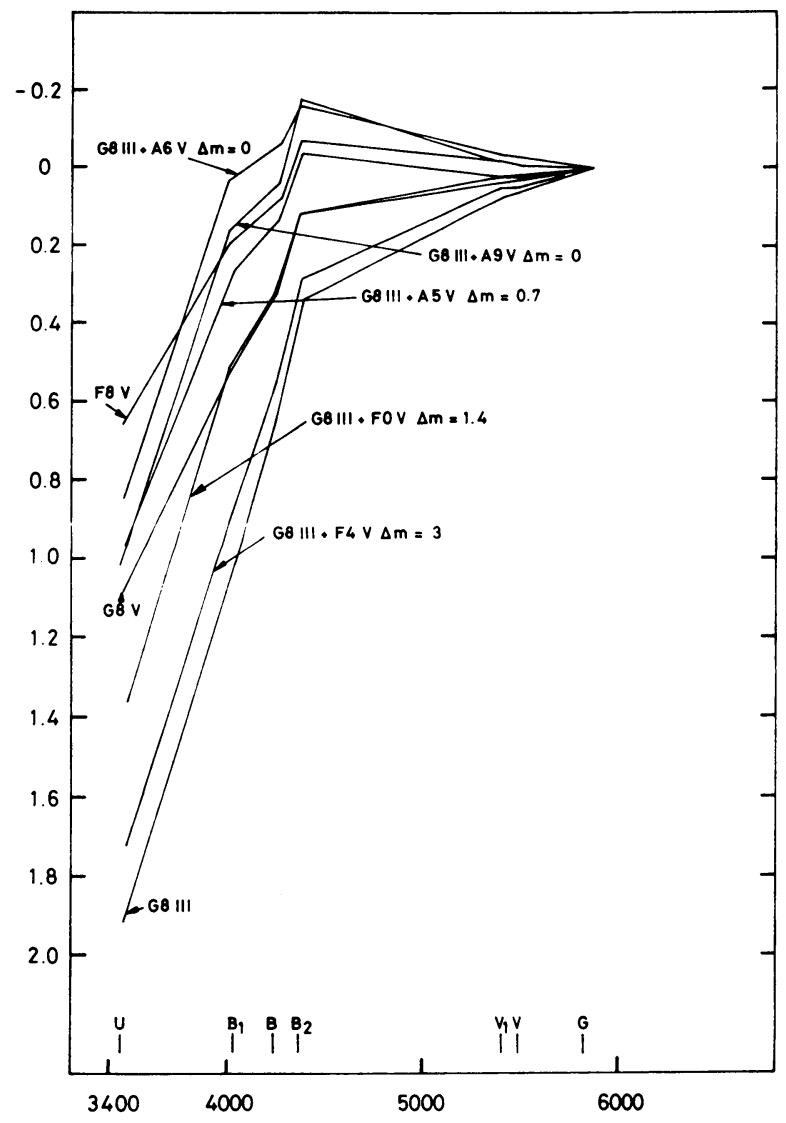

Fig. 2. Effect of binarity for some G8 III stars.

\section{Choice of the 'HR Standard' Stars}

The 'HR standard' stars are to be used for the calibration of mean relations, such as: $M_{v}$, colour indices;

$\theta_{\text {eff }}$, colour indices or combinations of colour indices;

Spectrum, colour indices;

$[\mathrm{Fe} / \mathrm{H}]$, colour indices or combinations of colour indices;

$\chi$, colour indices or combinations of colour indices;

etc.

The colours can be those obtained with wide bandpasses (example $U B V R I$ ), narrow bandpasses (example $u v b y$ ), intermediary bandpasses (example $U B_{1} B_{2} V_{1} G$ ).

The determination of $\theta_{\text {eff }}, \log g, \chi$ requires the fitting of stellar models to observed energy distributions or, at least, to the known colours of the star considered. This involves the necessity to know and to preserve the response functions of the photometric system with an accuracy equal to that of the intensity measurements. Thus, 


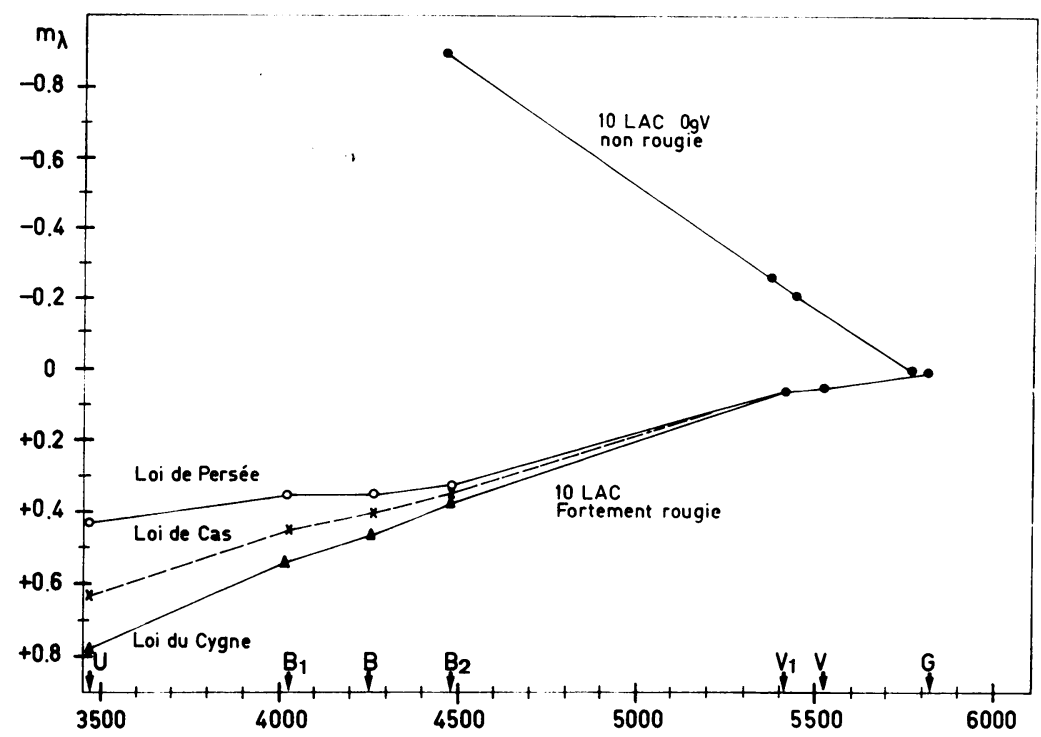

Fig. 3. Deformation of the pseudo-continuum for an $\mathrm{O}$ star resulting from three different laws.

these remarks imply that accurate photometric measurements of the stars proposed as 'HR standard' are available in a photometric system with well defined bandpasses. Moreover, the number of separate measurements of each star has to be sufficient to be able to detect any possible variability. The $U B V B_{1} B_{2} V_{1} G$ photometric system meets these conditions. This system has been in use at the Geneva Observatory since 1959. Its properties are described by Golay $(1969,1971,1972)$. The last catalogue published by Rufener (1971) contains more than 1500 stars, and will very soon reach 2500. This publication contains the response functions of the seven filters as well as a discussion of the accuracy of the measurements. Here, we have only considered stars with a weight $p \geqslant 3$, which corresponds to the following standard deviations expressed in thousandths of a magnitude (Table I). In Table I we give the standard deviations and the mean wavelengths of the bandpasses (whose equivalent rectangular bandpasses are between $300 \AA$ and $600 \AA$ ).

TABLE I

Mean wavelengths and standard deviations

\begin{tabular}{llllllll}
\hline & $U$ & $B$ & $V$ & $B_{1}$ & $B_{2}$ & $V_{1}$ & $G$ \\
\hline$\lambda_{0}[\AA]$ & 3456 & 4245 & 5500 & 4024 & 4480 & 5405 & 5805 \\
$\sigma$ & 6,1 & 3,2 & 3,9 & 3,2 & 3,3 & 3,7 & 4,4 \\
\hline
\end{tabular}

The stars we propose as 'HR standards' all fulfill the following conditions:

(1) Are not binaries, (except the interesting case with well separated components) or the two components are practically identical $(\Delta m \leqslant 0.1$ and same spectral type) or the difference in magnitude $\Delta m$ is $\geqslant 5$. 

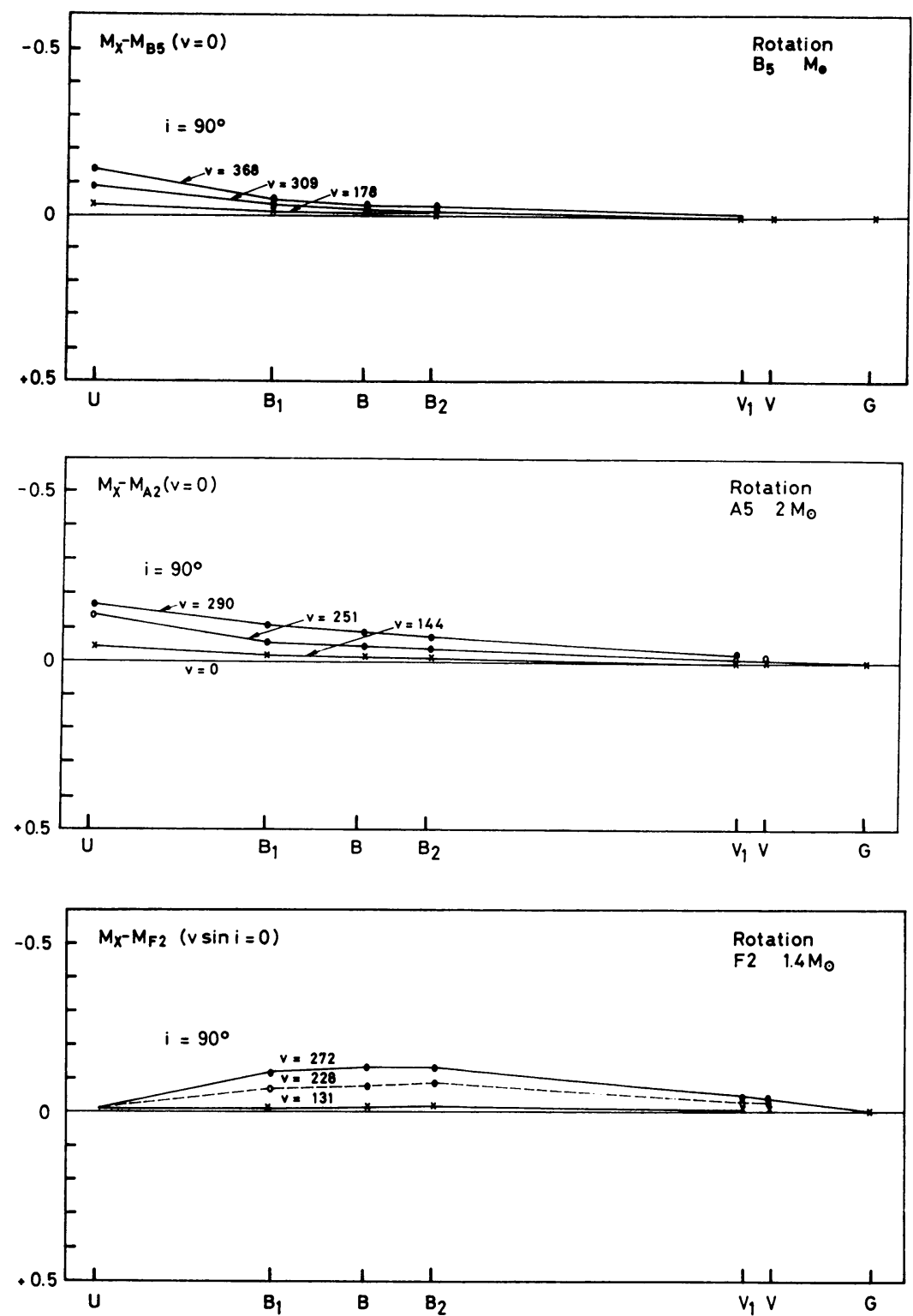

Fig. 4a, b, c. Deformation of the pseudo-continuum caused by stellar rotation (colours computed by Maeder with models of Maeder and Peytremann 1970, 1972).

(2) Are not, or are but slightly reddened by interstellar matter.

(3) Are not suspected to be variable. We have retained as HR standard, stars suspected of variability in Literature, but not confirmed as such in our photometry. We identify with asterisks those stars not known to be variable, but which our measurements lead us to suspect of being variable. 

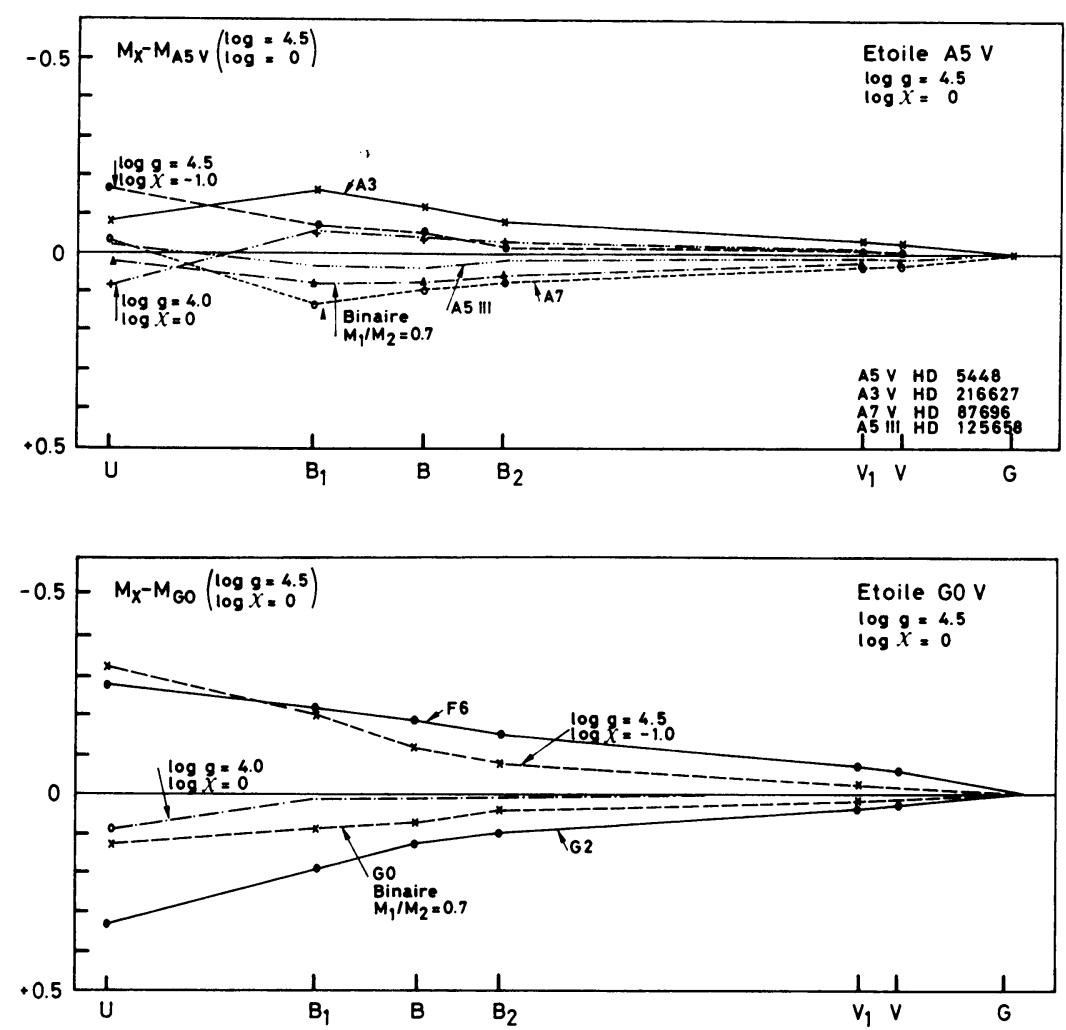

Fig. 5a, b. Comparison with help of models (Maeder and Peytremann) of the effects of binarity gravity, chemical composition $(\log \chi \simeq 0.6[\mathrm{Fe} / \mathrm{H}])$, spectral type, for two normal stars A5 V, G0 V.

(4) Have $V \sin i>150 \mathrm{~km} \mathrm{~s}^{-1}$ when this is known.

Moreover, they must belong to one or more of the following series:

(a) They must be among the MK standards published by Morgan and Roman (1950), Johnson and Morgan (1953), and Morgan et al. (1953).

(b) Be among the A stars classified MK by Cowley et al. (1969).

(c) Be among the stars having a trigonometric parallax of class A, B, C in Gliese's catalogue (1969).

(d) Be among stars which have once been the object of a study of abundances by means of spectroscopic observations. The stars have been selected from the lists of Cayrel and Cayrel de Strobel (1966) and Powell (1970).

(e) Be probable members of open clusters of known distance modulus. Our list is limited for the moment to the Hydaes, Praesepe and Coma Berenices clusters.

In the following pages, we give a serie of tables. Tables 1 to 7 contain all the stars we propose as HR standard. Each table contains stars belonging to the same original list. 
Table 1 contains the stars of series a

Table 2 contains the stars of series $b$

Table 3 contains the stars of series $c$

Table 4 contains the stars of series $d$

Table 5 members of the Hyades

Table 6 members of Praesepe

Table 7 members of Coma Berenices

TABLE 1

MK Standards

\begin{tabular}{|c|c|c|c|c|c|c|c|}
\hline $\mathrm{HD}$ or $\mathrm{BD}$ & $\begin{array}{l}\text { HR or } \\
\text { other }\end{array}$ & $\mathrm{Sp}$ & $m_{v}$ & $B_{2}-V_{1}$ & Variab. & $V \sin i$ & Remarks \\
\hline 14633 & & 08 & 7.459 & -0.285 & $* * *$ & 126 & \\
\hline 38771 & 2004 & B0.5 Ia & $(2.040)$ & -0.242 & $* *$ & 81 & $\beta y$ \\
\hline 163506 & 6685 & F2 Ia & 5.419 & 0.146 & $* * *$ & 23 & $\beta y$ \\
\hline 212593 & 8541 & B9 Iab & $(4.530)$ & -0.038 & & 29 & \\
\hline 91316 & 4133 & B1 Ib & 3.858 & -0.217 & & 69 & $\beta y$ \\
\hline 87737 & 3975 & AO Ib & 3.522 & -0.142 & & 18 & $\begin{array}{l}1,2 \\
\beta y\end{array}$ \\
\hline 46300 & 2385 & A0 Ib & $(4.480)$ & -0.116 & & 17 & $1,2, \beta y$ \\
\hline 20902 & 1017 & F5 Ib & 1.811 & 0.241 & $* *$ & 18 & $1,4, \beta y, \mathrm{DG}$ \\
\hline 164136 & 6707 & F2 II & 4.402 & 0.177 & & 27 & $1,4, \beta y$ \\
\hline 571 & 27 & F2 II & 5.030 & 0.199 & & 47 & By \\
\hline 35468 & 1790 & B2 III & 1.634 & -0.297 & & 64 & $1,8, \beta y$ \\
\hline 30836 & 1552 & B2 III & 3.667 & -0.248 & & 42 & $\beta y$ \\
\hline 22928 & 1122 & B5 III & 3.012 & -0.240 & & 271 & $1,8, \beta y$ \\
\hline 23302 & 1142 & B6 III & 3.704 & -0.215 & & 227 & \\
\hline 123299 & 5291 & A0 III & 3.659 & -0.180 & $* * *$ & 12 & $1,8, \beta y$ \\
\hline 89025 & 4031 & F0 III & $(3.430)$ & 0.105 & & 82 & By \\
\hline 13174 & 623 & F2 III & $(5.010)$ & 0.127 & & 154 & $\beta y$ \\
\hline 17584 & 840 & F2 III & $(4.220)$ & 0.141 & & 149 & $\beta y$ \\
\hline 21770 & 1069 & F4 III & 5.301 & 0.191 & & 29 & $\beta y$ \\
\hline 27022 & 1327 & G5 IIl & 5.269 & 0.524 & & $<19$ & $\beta y, \mathrm{DG}$ \\
\hline 28305 & 1409 & K0 III & 3.548 & 0.681 & & $\leqslant 8$ & $1,5, \mathrm{DG}$ \\
\hline 1013 & 45 & M2 III & 4.837 & 1.188 & $* * *$ & & 1,8 \\
\hline 47105 & 2421 & A0 IV & 1.939 & -0.151 & & 37 & $1,2, \beta y$ \\
\hline 211336 & 8494 & FO IV & 4.185 & 0.072 & & 86 & \\
\hline 17094 & 813 & F0 IV & 4.268 & 0.097 & & 54 & $\beta y$ \\
\hline 89449 & 4054 & F6 IV & 4.783 & 0.228 & & 16 & $\beta y$ \\
\hline 82328 & 3775 & F6 IV & 3.183 & 0.253 & $* * *$ & 13 & $1,4, \beta y$ \\
\hline 11443 & 544 & F6 IV & $(3.530)$ & 0.254 & & 95 & $1,4, \beta y$ \\
\hline 216385 & 8697 & F7 IV & $(5.220)$ & 0.261 & & 0 & By \\
\hline 220657 & 8905 & F8 IV & $(4.510)$ & 0.350 & & 79 & $\beta y$ \\
\hline 121370 & 5235 & G0 IV & 2.705 & 0.328 & & & By \\
\hline 23249 & 1136 & K0 IV & 3.548 & 0.601 & $* * *$ & $<17$ & $1,3,4,8, \mathrm{DG}$ \\
\hline 36512 & 1855 & B0 V & 4.592 & -0.320 & & 17 & By \\
\hline 3360 & 153 & B2 V & 3.646 & -0.276 & & 22 & $\beta y$ \\
\hline 74280 & 3454 & B3 V & 4.291 & -0.274 & & 132 & By \\
\hline 32630 & 1641 & B3 V & 3.155 & -0.270 & & 139 & $\beta y$ \\
\hline 83754 & 3849 & B5 V & $(5.060)$ & -0.250 & $* * *$ & 185 & $\beta y$ \\
\hline 23338 & 1145 & B6 V & 4.302 & -0.218 & & 134 & $\beta y$ \\
\hline
\end{tabular}


Table 1 (Continued)

\begin{tabular}{|c|c|c|c|c|c|c|c|}
\hline $\mathrm{HD}$ or $\mathrm{BD}$ & $\begin{array}{l}\text { HR or } \\
\text { other }\end{array}$ & $\mathrm{Sp}$ & $m_{v}$ & $B_{2}-V_{1}$ & Variab. & $V \sin i$ & Remarks \\
\hline 87901 & 3982 & B7 V & 1.375 & -0.219 & & 354 & $1,8, \beta y$ \\
\hline 214923 & 8634 & B8 V & 3.416 & -0.215 & & 196 & $\beta y$ \\
\hline 135742 & 5685 & B8 V & $(2.610)$ & -0.209 & * & 230 & $1,8, \beta y$ \\
\hline 222173 & 8965 & B8 V & 4.280 & -0.209 & * & 84 & By \\
\hline 103287 & 4554 & $\mathrm{~A} 0 \mathrm{~V}$ & 2.452 & -0.154 & & 163 & $1,2,8, \beta y$ \\
\hline 71155 & 3314 & A0 V & 3.902 & -0.159 & & 122 & $1,2,8, \beta y$ \\
\hline 139006 & 5793 & A0 V & (2.230) & -0.148 & & 132 & By \\
\hline 97633 & 4359 & A2 V & 3.320 & -0.154 & & 14 & $1,2,8, \beta y$ \\
\hline 1280 & 63 & A2 V & 4.608 & -0.113 & * & 107 & $1,2, \beta y$ \\
\hline 106591 & 4660 & A3 V & (3.313) & -0.089 & $* *$ & 177 & $1,2,3, \beta y$ \\
\hline 11636 & 553 & A5 V & 2.664 & -0.061 & & 73 & $1,8, \beta y$ \\
\hline 8538 & 403 & A5 V & 2.653 & -0.045 & $* *$ & 116 & $1,8, \beta y$ \\
\hline 87696 & 3974 & A7 V & 4.484 & -0.000 & & 157 & $1,2, \beta y$ \\
\hline 58946 & 2852 & FO V & 4.169 & 0.119 & & 63 & $1,3, \beta y$ \\
\hline 110379 & 4825 & FO V & 2.766 & 0.141 & * & 27 & $1,3,8, \beta y$ \\
\hline 91480 & 4141 & F1 V & 5.156 & 0.137 & & 79 & By \\
\hline 128167 & 5447 & F2 V & 4.474 & 0.168 & * & 0 & By \\
\hline 134083 & 5634 & F5 V & 4.930 & 0.210 & * & 44 & $1,3, \beta y$ \\
\hline 210027 & 8430 & F5 V & 3.770 & 0.216 & $* * *$ & 7 & $1,4, \beta y$ \\
\hline 30652 & 1543 & F6 V & 3.199 & 0.230 & & 16 & $1,3,4, \beta y$ \\
\hline 173667 & 7061 & F6 V & 4.194 & 0.249 & & 14 & $1,8, \beta y$ \\
\hline 142860 & 5933 & F6 V & 3.880 & 0.258 & $* *$ & 7 & $1,3,4,8, \beta y$ \\
\hline 120136 & 5185 & F7 V & 4.498 & 0.255 & & 14 & By \\
\hline 16895 & 799 & F7 V & 4.110 & 0.261 & $* * *$ & 6 & $1,4, \beta y$ \\
\hline 126660 & 5404 & F7 V & 4.051 & 0.264 & * & 31 & $1,3, \beta y$ \\
\hline 184960 & 7451 & F8 V & 5.719 & 0.252 & & $\leqslant 6$ & вy \\
\hline 90839 & 4112 & F8 V & 4.838 & 0.293 & & 0 & $1,3,4, \beta y$ \\
\hline 9826 & 458 & F8 V & $(4.080)$ & 0.294 & & 8 & $1,3,4, \beta y, \mathrm{DG}$ \\
\hline 102870 & 4540 & F8 V & 3.636 & 0.301 & & 0 & $1,3,4,8, \beta y$ \\
\hline 114710 & 4983 & GOV V & 4.252 & 0.332 & & 6 & $1,3,4, \beta y, \mathrm{DG}$ \\
\hline 19373 & 937 & G0V & 4.059 & 0.335 & & $\leqslant 10$ & $1,3,4, \beta y$, DG \\
\hline 13974 & 660 & G0 V & 4.873 & 0.351 & & $\leqslant 10$ & $1,3,4, \beta y, \mathrm{DG}$ \\
\hline 109358 & 4785 & G0 V & 4.276 & 0.350 & & $\leqslant 3$ & $1,3,4, \beta y, \mathrm{DG}$ \\
\hline 115043 & & G2 V & 6.811 & 0.356 & & $<50$ & $1,4, \beta y$ \\
\hline 10307 & 483 & $\mathrm{G} 2 \mathrm{~V}$ & 4.966 & 0.362 & & $\leqslant 3$ & $1,3,4, \beta y, \mathrm{DG}$ \\
\hline 20630 & 996 & G5 V & $(4.820)$ & 0.407 & & $<17$ & $1,4, \beta y, \mathrm{DG}$ \\
\hline 6582 & 321 & G5 Vp & 5.166 & 0.431 & $* *$ & $<17$ & $\beta y$, DG \\
\hline 117176 & 5072 & G5 V & 4.967 & 0.441 & & $\leqslant 10$ & $\beta y, \mathrm{DG}$ \\
\hline 10700 & 509 & G8 Vp & 3.481 & 0.424 & & $<17$ & $1,3, \beta y, \mathrm{DG}$ \\
\hline 101501 & 4496 & G8 V & 5.317 & 0.449 & * & $<17$ & $1,3, \mathrm{DG}$ \\
\hline 154345 & & G8 V & $(6.770)$ & 0.457 & $* *$ & & $1,3, \beta y, \mathrm{DG}$ \\
\hline 103095 & 4550 & G8 VI & 6.434 & 0.482 & & & $1,3,4, \beta y, \mathrm{DG}$ \\
\hline 10780 & 511 & KO V & 5.622 & 0.492 & & & $1,3, \mathrm{DG}$ \\
\hline 124752 & & $\mathrm{~K} 0 \mathrm{~V}$ & 8.517 & 0.519 & * & & \\
\hline 3651 & 166 & KO V & 5.900 & 0.526 & $* * *$ & & $1,3, \beta y, \mathrm{DG}$ \\
\hline 166620 & 6806 & $\mathrm{~K} 2 \mathrm{~V}$ & 6.393 & 0.552 & $* * *$ & & $\beta y$, DG \\
\hline 109011 & & $\mathrm{~K} 2 \mathrm{~V}$ & 8.103 & 0.599 & * & & DG \\
\hline 128165 & & K3 V & 7.255 & 0.616 & $* * *$ & & \\
\hline 219134 & 8832 & K3 V & $(5.570)$ & 0.628 & & & $1,3,4, \beta y, \mathrm{DG}$ \\
\hline 151288 & & K7 V & 8.091 & 0.919 & $* * *$ & & 1,3 \\
\hline
\end{tabular}


TABLE 2

Stars classified by $\mathrm{C}^{2} \mathrm{~J}^{2}$, Cowley et al. (1969)

\begin{tabular}{|c|c|c|c|c|c|c|c|}
\hline $\mathrm{HD}$ or $\mathrm{BD}$ & $\begin{array}{l}\text { HR or } \\
\text { other }\end{array}$ & $\mathrm{Sp}$ & $m_{v}$ & $B_{2}-V_{1}$ & Variab. & $V \sin i$ & Remarks \\
\hline 6457 & 311 & Ao Vn & 5.549 & -0.194 & & 267 & $\beta y$ \\
\hline 85504 & 3906 & A0 Vs & 6.018 & -0.185 & $* * *$ & & $\beta y$ \\
\hline 92728 & 4187 & A0 Vs & 5.794 & -0.185 & & 40 & \\
\hline 118214 & 5109 & AO V & 5.599 & -0.167 & $*$ & 197 & By \\
\hline 71155 & 3314 & $\mathrm{AO} \mathrm{V}$ & 3.902 & -0.159 & & 127 & $1,2,8, \beta y$ \\
\hline 23441 & 1152 & A0 Vn & 6.439 & -0.154 & $* *$ & 267 & \\
\hline 103287 & 4554 & A0 V & 2.452 & -0.154 & & 169 & $1,2,8, \beta y$ \\
\hline 6456 & 310 & A1 Vn & 5.329 & -0.150 & $* * *$ & 247 & $\beta y$ \\
\hline 2888 & 128 & $\mathrm{~A} 1 \mathrm{Vn}$ & 6.697 & -0.148 & & 265 & вy \\
\hline 14055 & 664 & Al Vnn & $(4.080)$ & -0.147 & & 225 & $\beta y$ \\
\hline 77327 & 3594 & A1 Vn & 3.584 & -0.147 & & 247 & $2,8, \beta y$ \\
\hline 30739 & 1544 & A1 V & 4.353 & -0.139 & & 250 & By \\
\hline 25490 & 1251 & A1 V & $(3.900)$ & -0.127 & & 110 & $2,8, \beta y$ \\
\hline 111397 & 4865 & $\mathrm{~A} 1 \mathrm{~V}$ & 5.707 & -0.125 & & & By \\
\hline 97633 & 4359 & A2 V & 3.320 & -0.155 & & 2 & $1,2,8, \beta y$ \\
\hline 50973 & 2585 & A2 Vn & $(4.890)$ & -0.129 & * & 210 & By \\
\hline 125642 & 5373 & A2 V & 6.308 & -0.115 & & & By \\
\hline 12471 & 599 & $\mathrm{~A} 2 \mathrm{~V}$ & 5.505 & -0.114 & & 100 & By \\
\hline 1280 & 63 & A2 V & 4.608 & -0.113 & * & 116 & $1,2, \beta y$ \\
\hline 28978 & 1448 & A2 Vs & 5.685 & -0.103 & & & By \\
\hline 146738 & 6074 & A3 V & 5.797 & -0.108 & $* * *$ & 80 & By \\
\hline 23848 & 1177 & A3 V & $(5.100)$ & -0.101 & & 117 & $\beta y$ \\
\hline 216627 & 8709 & A3 V & (3.290) & -0.096 & $* * *$ & 96 & $\beta y$ \\
\hline 27820 & 1381 & A3 V & 5.112 & -0.089 & & 90 & By \\
\hline 106591 & 4660 & A3 V & (3.313) & -0.088 & $* *$ & 179 & $1,2,3, \beta y$ \\
\hline 18331 & 875 & A3 V & $(5.170)$ & -0.084 & & 300 & By \\
\hline 141003 & 5867 & A3 V & 3.674 & -0.081 & & 200 & $\beta y$ \\
\hline 56537 & 2763 & A3 V & 3.585 & -0.076 & & 157 & By \\
\hline 14417 & 684 & A3 V & 6.480 & -0.069 & & & $\beta y$ \\
\hline 118098 & 5107 & A3 V & 3.373 & -0.068 & & 195 & By \\
\hline 119024 & 5142 & A3 Vn & 5.467 & -0.068 & & 215 & By \\
\hline 108382 & 4738 & A4 V & 4.986 & -0.075 & & 94 & 2,8 \\
\hline 105805 & 4633 & A4 Vn & 6.014 & -0.055 & $* *$ & 172 & 2,7 \\
\hline 38091 & 1969 & A4 Vn & 5.935 & -0.027 & $* * *$ & 260 & By \\
\hline 5448 & 269 & A5 V & $(3.930)$ & -0.052 & & 77 & By \\
\hline 116842 & 5062 & A5 V & 4.016 & -0.020 & & 250 & By \\
\hline 220061 & 8880 & A5 V & 4.589 & -0.005 & & 175 & $\beta y$ \\
\hline 79439 & 3662 & A5 V & 4.814 & 0.007 & & 178 & By \\
\hline 32301 & 1620 & A7 V & (4.640) & -0.036 & & 131 & $2,5, \beta y$ \\
\hline 177196 & 7215 & A7 V & $(5.000)$ & -0.009 & & 140 & $\beta y$ \\
\hline 87696 & 3974 & A7 V & 4.484 & 0.000 & & 168 & $1,2, \beta y$ \\
\hline 27084 & 1330 & A7 V & 4.454 & 0.040 & & 97 & \\
\hline 95608 & 4300 & A1 m: & 4.423 & -0.122 & & 24 & $\beta y$ \\
\hline 17581 & 839 & A1 m: & $(6.380)$ & -0.072 & & & By \\
\hline 195479 & 7839 & $\mathrm{~A} 1 \mathrm{~m}$ & 6.202 & -0.058 & & & By \\
\hline 78209 & 3619 & Al m & 4.463 & 0.069 & & 38 & By \\
\hline 36484 & 1850 & A2 m & 6.487 & -0.091 & & & By \\
\hline 12869 & 613 & A2 m: & 5.031 & -0.064 & & 0 & By \\
\hline 223461 & 9025 & $\mathrm{~A} 2 \mathrm{~m}$ & 5.968 & -0.020 & & & By \\
\hline 140232 & 5845 & $\mathrm{~A} 2 \mathrm{~m}$ & 5.795 & 0.003 & & & By \\
\hline
\end{tabular}


Table 2 (Continued)

\begin{tabular}{|c|c|c|c|c|c|c|c|}
\hline HD or BD & $\begin{array}{l}\text { HR or } \\
\text { other }\end{array}$ & Sp & $m_{v}$ & $B_{2}-V_{1}$ & Variab. & $V \sin i$ & Remarks \\
\hline 72037 & 3354 & A2 $\mathrm{m}$ & 5.452 & 0.003 & & 38 & $\beta y$ \\
\hline 166926 & 6811 & $\mathrm{~A} 2 \mathrm{~m}$ & $(5.820)$ & 0.018 & & & \\
\hline 33254 & 1672 & $\mathrm{~A} 2 \mathrm{~m}$ & 5.432 & 0.031 & & 25 & \\
\hline 99945 & 4429 & $\mathrm{~A} 2 \mathrm{~m}$ & 6.117 & 0.048 & $*$ & & \\
\hline 221675 & 8944 & A2 $\mathrm{m}$ & 5.886 & 0.081 & & & $\beta y$ \\
\hline 18769 & 905 & A3 m & $(5.860)$ & -0.049 & $* * *$ & 50 & $\beta y$ \\
\hline 79193 & 3655 & A3 $\mathrm{m}$ & 6.104 & 0.033 & & & By \\
\hline 141675 & 5887 & A3 $\mathrm{m}$ & 5.866 & 0.033 & & 82 & By \\
\hline 195217 & 7833 & A3 m & $(6.330)$ & 0.037 & & & By \\
\hline 27045 & 1329 & A3 m & 4.944 & 0.049 & & 60 & $\beta y$ \\
\hline 102660 & 4535 & A3 m & 6.045 & 0.063 & & & By \\
\hline 27628 & 1368 & A3 m & $(5.720)$ & 0.104 & & 15 & 2,5 \\
\hline 33641 & 1689 & A4 m: & (4.740) & -0.001 & & 84 & $\beta y$ \\
\hline 32428 & 1627 & $\mathrm{~A} 4 \mathrm{~m}$ & 6.602 & 0.070 & & & $\beta y$ \\
\hline 159560 & 6555 & A4 m & 4.867 & 0.070 & & 47 & By \\
\hline 76756 & 3572 & A5 $\mathrm{m}$ & 4.266 & -0.046 & $*$ & 74 & $\beta y$ \\
\hline 15385 & 723 & A5 $\mathrm{m}$ & 6.190 & -0.038 & & 60 & $\beta y$ \\
\hline 6116 & 290 & A5 $\mathrm{m}$ : & 5.955 & -0.024 & & & $\beta y$ \\
\hline 24141 & 1192 & A5 $\mathrm{m}$ : & 5.795 & -0.023 & & & $\beta y$ \\
\hline 60652 & 2914 & A5 m & 5.913 & 0.108 & & & $\beta y$ \\
\hline 111421 & 4866 & A6 m: & 6.250 & -0.012 & & 40 & By \\
\hline 3883 & 178 & $\mathrm{~A} 7 \mathrm{~m}$ & 6.059 & 0.046 & $*$ & & $\beta y$ \\
\hline 107168 & 4685 & A8 m: & 6.250 & -0.024 & & $\leqslant 12$ & \\
\hline 90569 & 4101 & A0 p & 6.013 & -0.207 & & 90 & $\beta y$ \\
\hline 10221 & 478 & A0 p & 5.569 & -0.205 & & 30 & $\beta y$ \\
\hline 32549 & 1638 & A0 p & 4.666 & -0.205 & & 29 & $\beta y$ \\
\hline 111133 & 4854 & A0 p & $(6.350)$ & -0.203 & & & $\beta y$ \\
\hline 4778 & 234 & A0 p & (6.140) & -0.173 & & & $\beta y$ \\
\hline 38104 & 1971 & A0 p & 5.467 & -0.135 & & 40 & $\beta y$ \\
\hline 74521 & 3465 & A1 p & 5.655 & -0.242 & & & $\beta y$ \\
\hline 72968 & 3398 & A1 p & 5.735 & -0.188 & & & $\beta y$ \\
\hline 151199 & 6226 & $A 2 p$ & 6.178 & -0.106 & & 110 & By \\
\hline 65339 & 3109 & $A 2 p$ & 6.035 & -0.057 & & & By \\
\hline 81009 & 3724 & A5 p & 6.518 & 0.001 & $* *$ & & $\beta y$ \\
\hline 87737 & 3975 & A0 Ib & 3.522 & -0.142 & & 29 & $1,2, \beta y$ \\
\hline 46300 & 2385 & A0 Ib & (4.480) & -0.115 & & 17 & $1,2, \beta y$ \\
\hline 196821 & 7903 & A0 III & (5.910) & -0.185 & $* * *$ & & By \\
\hline 221756 & 8947 & A1 III & (5.580) & -0.089 & $* *$ & 145 & $\beta y$ \\
\hline 50019 & 2540 & A3 III & 3.601 & -0.063 & & 140 & $\beta y$ \\
\hline 109307 & 4780 & A5 III & $(6.230)$ & -0.066 & & 8 & 2,7 \\
\hline 173880 & 7069 & A5 III & (4.300) & -0.048 & & 81 & $\beta y$ \\
\hline 125658 & 5374 & A5 III & 6.450 & -0.042 & $*$ & & $\beta y$ \\
\hline 47105 & 2421 & A1 IV & 1.939 & -0.151 & & 26 & $1,2, \beta y$ \\
\hline 89021 & 4033 & A2 IV & 3.434 & -0.115 & & 43 & By \\
\hline 107966 & 4717 & A3 IV & 5.163 & -0.085 & & 50 & 2,7 \\
\hline 28527 & 1427 & A6 IV & (4.780) & -0.019 & & 65 & \\
\hline 138341 & 5760 & A4 lV & 6.455 & 0.017 & & & $\beta y$ \\
\hline 27934 & 1387 & A7 IV-V & $(4.220)$ & -0.056 & & 77 & \\
\hline 107131 & 4684 & A7 IV-V & 6.423 & -0.022 & & 175 & 2,7 \\
\hline 203280 & 8162 & A7 IV-V & 2.461 & 0.019 & $*$ & 260 & $2,3, \beta y$ \\
\hline 21551 & 1051 & B8 Vnn & 5.819 & -0.163 & & 380 & \\
\hline
\end{tabular}


Table 2 (Continued)

\begin{tabular}{|c|c|c|c|c|c|c|c|}
\hline $\mathrm{HD}$ or $\mathrm{BD}$ & $\begin{array}{l}\text { HR or } \\
\text { other }\end{array}$ & Sp & $m_{v}$ & $B_{2}-V_{1}$ & Variab. & $V \sin i$ & Remarks \\
\hline 79469 & 3665 & B9.5 V & $(3.880)$ & -0.208 & & 91 & $2,8, \beta y$ \\
\hline 47964 & 2461 & B8 III & 5.793 & -0.208 & & 95 & \\
\hline 182691 & 7381 & B9 III & 6.493 & -0.192 & & & \\
\hline 205551 & 8259 & B9 III & (5.940) & -0.122 & & 200 & \\
\hline 35600 & 1804 & B9 Ib & 5.709 & 0.041 & & & \\
\hline 133029 & 5597 & B9 p & $(6.380)$ & -0.256 & & & $\beta y$ \\
\hline 205087 & 8240 & B9 p & 6.701 & -0.230 & * & & вy \\
\hline 68351 & 3215 & B9 p & 5.618 & -0.216 & & 0 & вy \\
\hline 207857 & 8349 & B9 p & $(6.160)$ & -0.195 & $* * *$ & & By \\
\hline 184961 & 7452 & B9 p? & 6.328 & -0.191 & $* *$ & 50 & $\beta y$ \\
\hline 145389 & 6023 & B9 $\mathrm{p}$ & 4.237 & -0.185 & & 0 & By \\
\hline 219749 & 8861 & B9 p & $(6.270)$ & -0.163 & & 70 & вy \\
\hline 148112 & 6117 & B9 p & $(4.560)$ & -0.156 & & 44 & $2,8, \beta y$ \\
\hline 173650 & 7058 & B9 p & $(6.500)$ & -0.137 & & & By \\
\hline 27176 & 1331 & Fo V & $(5.650)$ & 0.073 & & 105 & 2,5 \\
\hline 107326 & 4694 & F0 IV & $(6.080)$ & 0.099 & & 125 & \\
\hline 108283 & 4733 & FO IIInp & 4.922 & 0.085 & & 227 & \\
\hline 118295 & 5116 & FO III & 6.841 & 0.008 & & & $\beta y$ \\
\hline 126661 & 5405 & F0 m & 5.408 & 0.031 & & 50 & By \\
\hline 176232 & 7167 & F0 p & 5.915 & 0.038 & & 103 & By \\
\hline
\end{tabular}

TABLE 3

Stars with trigonometric parallax $A, B, C$

\begin{tabular}{|c|c|c|c|c|c|c|c|}
\hline HD or DB & $\begin{array}{l}\text { HR or } \\
\text { other }\end{array}$ & $\mathrm{Sp}$ & $m_{v}$ & $B_{2}-V_{1}$ & Variab. & $V \sin i$ & Remarks \\
\hline $63^{\circ} 0137$ & & K7 V & 8.983 & 0.873 & $* *$ & & $\begin{array}{l}8.29 \\
\mathrm{DG}\end{array}$ \\
\hline 166 & 8 & $\mathrm{~K} 0 \mathrm{~V}$ & 6.085 & 0.462 & $*$ & & $\begin{array}{l}5.3 \\
3, \mathrm{DG}\end{array}$ \\
\hline 3651 & 166 & K0 V & 5.900 & 0.526 & $* * *$ & & $\begin{array}{l}5.75 \\
\beta y, \text { DG, } 1\end{array}$ \\
\hline 4628 & 222 & K2 V & $(5.760)$ & 0.549 & & & $\begin{array}{l}6.55 \\
3,8, \mathrm{DG}\end{array}$ \\
\hline 9826 & 458 & F8 V & $(4.080)$ & 0.294 & & 8 & $\begin{array}{l}3.06 \\
1,3,4, \beta y, \mathrm{DG}\end{array}$ \\
\hline 10307 & 483 & G2 V & 4.966 & 0.362 & & $\leqslant 3$ & $\begin{array}{l}4.66 \\
1,3,4, \beta y, \mathrm{DG}\end{array}$ \\
\hline 10700 & 509 & G8 Vp & 3.481 & 0.424 & & $<17$ & $\begin{array}{l}5.72 \\
1,3, \beta y, \text { DG }\end{array}$ \\
\hline 10780 & 511 & $\mathrm{~K} 0 \mathrm{~V}$ & 5.622 & 0.492 & & & $\begin{array}{l}5.91 \\
1,3, \mathrm{DG}\end{array}$ \\
\hline 13974 & 660 & G0 V & 4.873 & 0.351 & & $\leqslant 10$ & $\begin{array}{l}4.80 \\
1,3,4, \beta y, \mathrm{DG}\end{array}$ \\
\hline 17925 & 857 & $\mathrm{~K} 0 \mathrm{~V}$ & 6.057 & 0.542 & $* *$ & & $\begin{array}{l}6.57 \\
\text { DG }\end{array}$ \\
\hline 19373 & 937 & G0 V & 4.059 & 0.335 & & $\leqslant 10$ & $\begin{array}{l}3.72 \\
1,3,4, \beta y, \mathrm{DG}\end{array}$ \\
\hline
\end{tabular}


Table 3 (Continued)

\begin{tabular}{|c|c|c|c|c|c|c|c|}
\hline $\mathrm{HD}$ or $\mathrm{BD}$ & $\begin{array}{l}\text { HR or } \\
\text { other }\end{array}$ & Sp & $m_{v}$ & $B_{2}-V_{1}$ & Variab. & $V \sin i$ & Remarks \\
\hline 23249 & & KO IV & 3.548 & 0.601 & $* * *$ & $<17$ & $\begin{array}{l}3.77 \\
1,3,4,8, \mathrm{DG}\end{array}$ \\
\hline 25329 & & K1 Vsd & 8.495 & 0.561 & $* *$ & & $3,4, \beta y$, DG \\
\hline 25680 & 1262 & G5 V & 5.900 & 0.368 & & & 5.09 \\
\hline 30652 & 1543 & F6 V & 3.199 & 0.230 & & 16 & $\begin{array}{l}3.76 \\
1,3,4, \beta y\end{array}$ \\
\hline 34411 & 1729 & G0 V & 4.706 & 0.360 & & $\leqslant 3$ & $3,4, \beta y$, DG \\
\hline 37394 & 1925 & K1 V & 6.224 & 0.524 & & & $\begin{array}{l}6.07 \\
\text { DG }\end{array}$ \\
\hline 58946 & 2852 & F0 V & 4.169 & 0.119 & & 63 & $\begin{array}{l}2.84 \\
1,3, \beta y\end{array}$ \\
\hline 65583 & & G8 V & $(7.000)$ & 0.451 & & & $\begin{array}{l}5.82 \\
\beta y, \mathrm{DG}\end{array}$ \\
\hline 72905 & 3391 & G0 V & 5.629 & 0.362 & & 4 & $\begin{array}{l}4.67 \\
\beta y, \mathrm{DG}\end{array}$ \\
\hline 76644 & 3569 & A7 V & 3.127 & 0.015 & & 138 & $\begin{array}{l}2.24 \\
\beta y\end{array}$ \\
\hline 84035 & & K5 V & 8.139 & 0.712 & $* * *$ & & 7.31 \\
\hline 84737 & 3881 & G1 V & 5.089 & 0.362 & & $\leqslant 10$ & $\begin{array}{l}4.20 \\
\text { By }\end{array}$ \\
\hline 88230 & & K7 V & 6.597 & 0.931 & $* * *$ & & $\begin{array}{l}8.32 \\
\beta y, \mathrm{DG}\end{array}$ \\
\hline 89125 & 4039 & DF3 & 5.820 & 0.279 & & $\leqslant 6$ & $\begin{array}{l}4.7 \\
\text { By }\end{array}$ \\
\hline 90839 & 4112 & F8 V & 4.838 & 0.293 & & 0 & $\begin{array}{l}4.44 \\
1,3,4, \beta y\end{array}$ \\
\hline 95128 & 4277 & Go V & 5.043 & 0.364 & & $\leqslant 3$ & $\begin{array}{l}4.4 \\
\beta y, \mathrm{DG}\end{array}$ \\
\hline 101501 & 4496 & G8 V & 5.317 & 0.449 & $*$ & $<17$ & $\begin{array}{l}5.55 \\
1,3, \mathrm{DG}\end{array}$ \\
\hline 102870 & 4540 & F8 V & 3.636 & 0.301 & & 0 & $\begin{array}{l}3.60 \\
1,3,4,8, \beta y\end{array}$ \\
\hline 103095 & 4550 & G8 VI & 6.434 & 0.482 & & & $\begin{array}{l}6.71 \\
1,3,4, \beta y, \mathrm{DG}\end{array}$ \\
\hline 106591 & 4660 & A3 V & (3.313) & -0.089 & $* *$ & 177 & 1.9 \\
\hline 109358 & 4785 & G0 V & 4.276 & 0.350 & & $\leqslant 3$ & $\begin{array}{l}4.46 \\
1,3,4, \beta y, \mathrm{DG}\end{array}$ \\
\hline 110379 & 4825 & F0 V & 2.766 & 0.141 & $*$ & 27 & $\begin{array}{l}3.46 \\
1,3,8, \beta y\end{array}$ \\
\hline 110833 & & K3 V & 7.017 & 0.585 & & & $\begin{array}{l}6.0 \\
\text { DG }\end{array}$ \\
\hline 110897 & 4845 & G0 V & 5.961 & 0.329 & & $\leqslant 6$ & $\begin{array}{l}5.0 \\
3,4, \beta y, \text { DG }\end{array}$ \\
\hline 114710 & & G0 V & 4.252 & 0.332 & & 6 & $\begin{array}{l}4.66 \\
1,3,4, \beta y, \mathrm{DG}\end{array}$ \\
\hline 126660 & 5404 & F7 V & 4.051 & 0.264 & $*$ & 31 & $\begin{array}{l}3.22 \\
1,3, \beta y\end{array}$ \\
\hline 131156 & 5544 & G8 V & 4.555 & 0.483 & & $<16$ & $\begin{array}{l}\text { A: } 5.53 \mathrm{~B}: \\
7.69\end{array}$ \\
\hline
\end{tabular}


Table 3 (Continued)

\begin{tabular}{|c|c|c|c|c|c|c|c|}
\hline $\mathrm{HD}$ or $\mathrm{BD}$ & $\begin{array}{l}\text { HR or } \\
\text { other }\end{array}$ & Sp & $m_{v}$ & $B_{2}-V_{1}$ & Variab. & $V \sin i$ & Remarks \\
\hline 131511 & 5553 & $\mathrm{~K} 2 \mathrm{~V}$ & 6.017 & 0.528 & * & & $\begin{array}{l}5.66 \\
\text { DG }\end{array}$ \\
\hline 134083 & 5634 & F5 V & 4.930 & 0.210 & $*$ & 44 & $\begin{array}{l}3.9 \\
1,3, \beta y\end{array}$ \\
\hline 139323 & & K3 V & 7.654 & 0.581 & $*$ & & 6.2 \\
\hline 142860 & 5933 & F6 V & 3.880 & 0.258 & $* *$ & 7 & $\begin{array}{l}3.4 \\
1,3,4,8, \beta y\end{array}$ \\
\hline 145417 & & KO V & 7.521 & 0.514 & & & 6.64 \\
\hline 145675 & & KO V & 6.611 & 0.542 & & & $\begin{array}{l}5.6 \\
\text { DG }\end{array}$ \\
\hline 151288 & & K7 V & 8.091 & 0.919 & $* * *$ & & $\begin{array}{l}8.19 \\
1,3\end{array}$ \\
\hline 154345 & & G8 V & $(6.770)$ & 0.457 & $* *$ & & $\begin{array}{l}5.73 \\
1,3, \beta y, \mathrm{DG}\end{array}$ \\
\hline 157214 & 6458 & G8 V & 5.388 & 0.381 & $*$ & 0 & $\begin{array}{l}4.71 \\
\beta y, \mathrm{DG}\end{array}$ \\
\hline 160346 & & K3 V & 6.529 & 0.606 & & & $\begin{array}{l}6.06 \\
\text { DG }\end{array}$ \\
\hline 182488 & 7368 & KO V & $(6.380)$ & 0.504 & & & $\begin{array}{l}5.14 \\
\text { DG }\end{array}$ \\
\hline 190406 & 7672 & G IV & 5.795 & 0.363 & $* * *$ & 4 & $\begin{array}{l}4.6 \\
\beta y, \mathrm{DG}\end{array}$ \\
\hline 193664 & 7783 & G5 V & 5.905 & 0.345 & & & 5.07 \\
\hline 203280 & 8162 & A7 V-IV & 2.461 & 0.018 & * & 240 & $\begin{array}{l}1.5 \\
2,3, \beta y\end{array}$ \\
\hline $\begin{array}{l}217987 \\
219134\end{array}$ & 8832 & $\begin{array}{l}\text { M2 V } \\
\text { K3 V }\end{array}$ & $\begin{array}{c}7.356 \\
(5.570)\end{array}$ & $\begin{array}{l}1.131 \\
0.628\end{array}$ & & & $\begin{array}{l}9.59 \\
6.41 \\
1,3,4, \beta y, \text { DG }\end{array}$ \\
\hline
\end{tabular}

TABLE 4.1

Stars with abundances determinations, Cayrel and Cayrel de Strobel

\begin{tabular}{|c|c|c|c|c|c|c|c|}
\hline $\mathrm{HD}$ or $\mathrm{BD}$ & $\begin{array}{l}\text { HR or } \\
\text { others }\end{array}$ & Sp & $m_{v}$ & $B_{2}-V_{1}$ & Variab. & $V \sin i$ & Remarks \\
\hline 3627 & 165 & K3 III & $(3.210)$ & 0.898 & & $<17$ & $\begin{array}{l}0.00 \\
\text { DG }\end{array}$ \\
\hline 5015 & 244 & F8 IV-V & $(4.860)$ & 0.306 & & 6 & $\begin{array}{l}-0.15 \\
\beta y\end{array}$ \\
\hline 5544 & & K0 III & $(7.710)$ & 0.783 & & & 0.33 \\
\hline 10307 & 483 & G2 V & 4.966 & 0.362 & & $\leqslant 3$ & $\begin{array}{l}0.20 \\
1,3,4, \beta y . \mathrm{DG}\end{array}$ \\
\hline 10380 & 489 & K3 III & 4.466 & 0.995 & & $<19$ & $\begin{array}{l}0.00 \\
\text { DG }\end{array}$ \\
\hline 11443 & 544 & F6 IV & $(3.530)$ & 0.254 & & 95 & $\begin{array}{l}-0.12 \\
1,4, \beta y\end{array}$ \\
\hline $37^{\circ} 00432$ & & K2 III & $(9.010)$ & 0.680 & $* * *$ & & $\begin{array}{l}-0.25 \\
\mathrm{DG}, \beta y\end{array}$ \\
\hline
\end{tabular}


Table 4.1 (Continued)

\begin{tabular}{|c|c|c|c|c|c|c|c|}
\hline $\mathrm{HD}$ or $\mathrm{BD}$ & $\begin{array}{l}\text { HR or } \\
\text { others }\end{array}$ & Sp & $m_{v}$ & $B_{2}-V_{1}$ & Variab. & $V \sin i$ & Remarks \\
\hline 13974 & 660 & Go V & 4.873 & 0.351 & & $\leqslant 10$ & $\begin{array}{l}-0.43 \\
-0.18 \\
-0.51 \\
1,3,4, \beta y, \mathrm{DG}\end{array}$ \\
\hline 16895 & 799 & F7 V & 4.110 & 0.261 & $* * *$ & 6 & $\begin{array}{l}+0.07 \\
-0.02 \\
1,4, \beta y\end{array}$ \\
\hline 18474 & 885 & G4p & 5.484 & 0.594 & & & $\begin{array}{l}0.15 \\
\text { DG }\end{array}$ \\
\hline 19373 & 937 & G0 V & 4.059 & 0.335 & & $\leqslant 10$ & $\begin{array}{l}0.14 \\
0.26 \\
1,3,4, \beta y, \mathrm{DG}\end{array}$ \\
\hline 19445 & & F7 VI & 8.041 & 0.269 & $* * *$ & & $\begin{array}{l}-0.77 \\
-1.75 \\
-1.75 \\
\beta y\end{array}$ \\
\hline 20630 & 996 & G5 V & $(4.820)$ & 0.407 & & $<17$ & $\begin{array}{l}0.38 \\
1,4, \beta y, \mathrm{DG}\end{array}$ \\
\hline 20902 & 1017 & F5 lb & 1.811 & 0.241 & $* *$ & 18 & $\begin{array}{l}-0.45 \\
1,4, \beta y, \mathrm{DG}\end{array}$ \\
\hline 22484 & 1101 & F8 V & 4.288 & 0.335 & & 0 & 0.37 \\
\hline 22879 & & F9 V & 6.673 & 0.316 & & & $\begin{array}{l}-0.57 \\
\beta y\end{array}$ \\
\hline 23230 & 1135 & F5 II & $(3.770)$ & 0.209 & & 44 & $\begin{array}{l}-0.19 \\
\beta y, \mathrm{DG}\end{array}$ \\
\hline 23249 & 1136 & K0 IV & 3.548 & 0.601 & $* * *$ & $<17$ & $\begin{array}{l}0.00 \\
-0.09 \\
1,3,4,8, \mathrm{DG}\end{array}$ \\
\hline 25329 & & K1 V & 8.495 & 0.561 & $* *$ & & $\begin{array}{l}-2.30 \\
3,4, \beta y, \mathrm{DG}\end{array}$ \\
\hline 30455 & & G2 V & 6.954 & 0.374 & $* *$ & & $\begin{array}{l}-0.09 \\
-0.26 \\
\text { DG }\end{array}$ \\
\hline 30649 & & G1 V-VI & 6.959 & 0.355 & & & $\begin{array}{l}-0.32 \\
-0.20 \\
\beta y, \mathrm{DG}\end{array}$ \\
\hline 30652 & 1543 & F6 V & 3.199 & 0.230 & & & $\begin{array}{l}-0.40 \\
1,3,4, \beta y\end{array}$ \\
\hline 31398 & 1577 & K3 II & 2.707 & 1.149 & & $<17$ & $\begin{array}{l}0.00 \\
\beta y, \mathrm{DG}\end{array}$ \\
\hline 34411 & 1729 & G0 IV & 4.706 & 0.360 & & $\leqslant 3$ & $\begin{array}{l}0.14 \\
0.22 \\
3,4, \beta y, \mathrm{DG}\end{array}$ \\
\hline 55575 & 2721 & G0 V & 5.554 & 0.342 & & $\leqslant 6$ & $\begin{array}{l}-0.21 \\
\beta y, \mathrm{DG}\end{array}$ \\
\hline 64491 & 3083 & Ap & 6.224 & 0.082 & & 70 & -0.70 \\
\hline 72324 & 3369 & G9 III & 6.353 & 0.688 & & & $\begin{array}{l}0.32 \\
\text { DG }\end{array}$ \\
\hline 73665 & 3427 & K0 III & 6.396 & 0.648 & $*$ & 0 & $\begin{array}{l}-0.04 \\
\mathrm{DG}, 4,6, \beta y\end{array}$ \\
\hline 73710 & 3428 & K0 III & 6.418 & 0.671 & & $<45$ & -0.17 \\
\hline
\end{tabular}


Table 4.1 (Continued)

\begin{tabular}{|c|c|c|c|c|c|c|c|}
\hline $\mathrm{HD}$ or $\mathrm{BD}$ & $\begin{array}{l}\text { HR or } \\
\text { others }\end{array}$ & Sp & $m_{v}$ & $B_{2}-V_{1}$ & Variab. & $V \sin i$ & Remarks \\
\hline 82328 & 3775 & F6 IV & 3.183 & 0.253 & $* * *$ & 13 & $\begin{array}{l}\text { DG, } \beta y \\
-0.44 \\
1,4, \beta y\end{array}$ \\
\hline 86728 & 3951 & G4 V & 5.390 & 0.398 & & $\leqslant 10$ & $\begin{array}{l}0.34 \\
0.34 \\
\beta y, \text { DG }\end{array}$ \\
\hline 90508 & 4098 & G1 V & 6.435 & 0.366 & & $\leqslant 10$ & $\begin{array}{l}-0.23 \\
\beta y, \mathrm{DG}\end{array}$ \\
\hline 90839 & 4112 & F8 V & 4.838 & 0.293 & & 0 & $\begin{array}{l}0.23 \\
1,3,4, \beta y\end{array}$ \\
\hline 102870 & 4540 & F8 V & 3.636 & 0.301 & & 0 & $\begin{array}{l}0.33 \\
1,3,4,8, \beta y\end{array}$ \\
\hline 103095 & 4550 & G8 VI & 6.434 & 0.482 & & & $\begin{array}{l}-1.50 \\
1,3,4, \beta y, \text { DG }\end{array}$ \\
\hline 106516 & 4657 & F6 V & $(6.110)$ & 0.234 & & 8 & $\begin{array}{l}(+0.05) \\
(-0.86) \\
\beta y\end{array}$ \\
\hline 109358 & 4785 & G0 V & 4.276 & 0.350 & & $\leqslant 3$ & $\begin{array}{l}0.02 \\
1,3,4, \beta y, \mathrm{DG}\end{array}$ \\
\hline 109995 & & $\mathrm{~A} 0 \mathrm{~V}$ & 7.589 & -0.098 & & 30 & $\begin{array}{l}-1.20 \\
\beta y\end{array}$ \\
\hline 110897 & 4845 & G8 V & 5.961 & 0.329 & & $\leqslant 6$ & $\begin{array}{l}-0.32 \\
3,4, \beta y, \text { DG }\end{array}$ \\
\hline 114710 & 4983 & Go V & 4.252 & 0.332 & & 6 & $\begin{array}{l}0.19 \\
0.05 \\
0.08 \\
1,3,4, \beta y, \mathrm{DG}\end{array}$ \\
\hline 114762 & & F9 V & 7.302 & 0.311 & $* * *$ & & -0.59 \\
\hline 115043 & & G0 V & 6.811 & 0.356 & & $<50$ & $\begin{array}{l}-0.06 \\
-0.14 \\
1,4, \beta y\end{array}$ \\
\hline 122563 & 5270 & Pop II gi. & 6.177 & 0.642 & $*$ & & $\begin{array}{l}-2.90 \\
-2.65 \\
\beta y, \text { DG }\end{array}$ \\
\hline 142267 & 5911 & G2 V & 6.087 & 0.359 & $* *$ & & $\begin{array}{l}-0.28 \\
\beta y\end{array}$ \\
\hline 142860 & 5933 & F6 IV & 3.880 & 0.258 & $* *$ & 7 & $\begin{array}{l}-0.40 \\
-0.36 \\
1,3,4,8\end{array}$ \\
\hline 152792 & & Go V & 6.822 & 0.388 & & & $\begin{array}{l}-0.45 \\
\beta y, \mathrm{DG}\end{array}$ \\
\hline 157089 & & G0 V & $(6.960)$ & 0.333 & & & $\begin{array}{l}-0.57 \\
\beta y, \mathrm{DG}\end{array}$ \\
\hline 160693 & & G0 V & 8.381 & 0.336 & $* * *$ & & $\begin{array}{l}-0.69 \\
\text { DG }\end{array}$ \\
\hline 161817 & & A2 VI & 6.963 & -0.008 & $* * *$ & & $\begin{array}{l}(-0.41) \\
\beta y\end{array}$ \\
\hline 164136 & 6707 & F2 III & 4.402 & 0.177 & & 27 & $\begin{array}{l}0.08 \\
1,4, \beta y\end{array}$ \\
\hline 170153 & 6927 & F7 V & 3.546 & 0.279 & $* *$ & 11 & $\begin{array}{l}-0.64 \\
4,8, \beta y\end{array}$ \\
\hline 185657 & 7477 & G6 V & $(6.350)$ & 0.677 & & & -0.51 \\
\hline
\end{tabular}


Table 4.1 (Continued)

\begin{tabular}{|c|c|c|c|c|c|c|c|}
\hline HD or BD & $\begin{array}{l}\text { HR or } \\
\text { others }\end{array}$ & Sp & $m_{v}$ & $B_{2}-V_{1}$ & Variab. & $V \sin i$ & Remarks \\
\hline 187923 & 7569 & G2 V & $(6.154)$ & 0.389 & $* *$ & $\leqslant 10$ & $\begin{array}{l}0.12 \\
0.00 \\
\beta y, \text { DG }\end{array}$ \\
\hline 190404 & & $\mathrm{~K} 2 \mathrm{~V}$ & 7.288 & 0.502 & $*$ & & $\begin{array}{l}-0.20 \\
\text { DG }\end{array}$ \\
\hline 191046 & & K0 III & $(7.200)$ & 0.821 & & & $\begin{array}{l}-0.42 \\
D G\end{array}$ \\
\hline 193370 & 7770 & F5 Ib & $(5.220)$ & 0.401 & & 13 & $\begin{array}{l}-0.19 \\
\beta y\end{array}$ \\
\hline 197461 & 7928 & A7 III & 4.443 & 0.091 & $* *$ & 41 & $\begin{array}{l}0.50 \\
\beta y\end{array}$ \\
\hline 198149 & 7957 & KO IV & $(3.430)$ & 0.605 & & $<17$ & $\begin{array}{l}0.00 \\
\beta y, \mathrm{DG}\end{array}$ \\
\hline 201626 & & K0 IIIp & 8.121 & 0.823 & $* * *$ & & $\begin{array}{l}-1.45 \\
\beta y, \mathrm{DG}\end{array}$ \\
\hline 210027 & 8430 & F5 V & 3.770 & 0.216 & $* * *$ & 7 & $\begin{array}{l}-0.10 \\
1,4, \beta y\end{array}$ \\
\hline 215648 & 8665 & F7 V & $(4.190)$ & 0.265 & & 7 & $\begin{array}{l}-0.05 \\
\beta y\end{array}$ \\
\hline 218804 & 8825 & F5 IV & $(5.950)$ & 0.224 & & 18 & $-0.21, \beta y$ \\
\hline 219134 & 8832 & K3 V & (5.570) & 0.628 & & & $\begin{array}{l}0.00 \\
1,3,4, \beta y, \mathrm{DG}\end{array}$ \\
\hline 221170 & & Pop II gi. & 7.677 & 0.803 & $* *$ & & $\begin{array}{l}-2.70 \\
\beta y, \mathrm{DG}\end{array}$ \\
\hline 221345 & 8930 & G8 III & $(5.220)$ & 0.705 & $*$ & $<19$ & $\begin{array}{l}-0.20 \\
\text { DG }\end{array}$ \\
\hline 224930 & 9088 & G3 V & $(5.760)$ & 0.420 & $*$ & $\leqslant 6$ & $\begin{array}{l}-0.59 \\
-0.70 \\
-0.55 \\
-0.60, \beta y, \\
\text { DG }\end{array}$ \\
\hline
\end{tabular}

TABLE 4.2

Stars with abundances determinations, Powell

\begin{tabular}{|c|c|c|c|c|c|c|c|}
\hline $\mathrm{HD}$ or $\mathrm{BD}$ & $\begin{array}{l}\text { HR or } \\
\text { other }\end{array}$ & $\mathrm{Sp}$ & $m_{v}$ & $B_{2}-V_{1}$ & Variab. & $V \sin i$ & Remarks \\
\hline 9826 & 458 & F8 V & $(4.080)$ & 0.294 & & 8 & $\begin{array}{l}-0.11 \\
1,3,4, \beta y, \text { DG }\end{array}$ \\
\hline 19373 & 937 & G0 V & 4.059 & 0.335 & & $\leqslant 10$ & $\begin{array}{l}0.05 \\
\beta y, \text { DG }\end{array}$ \\
\hline 30652 & 1543 & F6 V & 3.199 & 0.230 & & & $\begin{array}{l}0.18 \\
\text { By }\end{array}$ \\
\hline 82328 & 3775 & F6 IV & 3.183 & 0.253 & $* * *$ & 13 & -0.03 \\
\hline 102870 & 4540 & F8 V & 3.636 & 0.301 & & 0 & $\begin{array}{l}0.15 \\
1,3,4,8, \beta y\end{array}$ \\
\hline 109358 & 4785 & G0 V & 4.276 & 0.350 & & $\leqslant 3$ & $\begin{array}{l}-0.23 \\
\beta y, \text { DG }\end{array}$ \\
\hline
\end{tabular}


Table 4.2 (Continued)

\begin{tabular}{|c|c|c|c|c|c|c|c|}
\hline HD or BD & $\begin{array}{l}\text { HR or } \\
\text { other }\end{array}$ & Sp & $m_{v}$ & $B_{2}-V_{1}$ & Variab. & $V \sin i$ & Remarks \\
\hline 136202 & 5694 & F8 IV-V & $(5.060)$ & 0.299 & & 0 & $\begin{array}{l}-0.17 \\
\beta y\end{array}$ \\
\hline 142373 & 5914 & F9 V & 4.614 & 0.340 & & 0 & -0.35 \\
\hline 142860 & 5933 & F6 IV & 3.880 & 0.258 & $* *$ & 7 & $\begin{array}{l}-0.11 \\
1,3,4,8, \beta y\end{array}$ \\
\hline 222368 & 8969 & F7 V & 4.130 & 0.269 & $* *$ & 6 & $\begin{array}{l}0.09 \\
\text { By }\end{array}$ \\
\hline
\end{tabular}

TABLE 5

Stars members of the Hyades

\begin{tabular}{|c|c|c|c|c|c|c|c|}
\hline $\mathrm{HD}$ or $\mathrm{BD}$ & $\begin{array}{l}\text { HR or } \\
\text { other }\end{array}$ & $\mathrm{Sp}$ & $m_{v}$ & $B_{2}-V_{1}$ & Variab. & $V \sin i$ & Remarks \\
\hline 27176 & 1331 & A8 V & $(5.650)$ & 0.073 & & 97 & $2,5, \beta y$ \\
\hline 27397 & 1351 & F3 V & $(5.590)$ & 0.068 & & 109 & By \\
\hline 27459 & 1356 & F0 V & 5.280 & 0.025 & & 65 & вy \\
\hline 27524 & & (F8) & $(6.800)$ & 0.203 & & 94 & By \\
\hline 27628 & 1368 & $\mathrm{Am}$ & $(5.720)$ & 0.104 & & 15 & $2,5, \beta y$ \\
\hline 27946 & 1388 & A7 V & $(5.280)$ & 0.048 & & 153 & By \\
\hline 28294 & 1408 & (F0) & 5.916 & 0.119 & & 102 & $\beta y$ \\
\hline 28305 & 1409 & K0 III & 3.548 & 0.680 & & $\leqslant 8$ & $1,5, \beta y, \mathrm{DG}$ \\
\hline 28406 & & (F8) & 6.899 & 0.231 & & 20 & By \\
\hline 28527 & 1427 & A7 V & $(4.780)$ & -0.019 & & 69 & By \\
\hline 28546 & 1428 & Am & 5.499 & 0.043 & & 23 & By \\
\hline 28556 & 1430 & (F1) & (5.400) & 0.048 & & 95 & By \\
\hline 28568 & & (F2) & (6.510) & 0.209 & & 53 & $\beta y$ \\
\hline 30780 & 1547 & A5 & $(5.100)$ & 0.022 & & 141 & By \\
\hline 32301 & 1620 & A7 V & (4.640) & -0.036 & & 127 & $2,5, \beta y$ \\
\hline
\end{tabular}

TABLE 6

Stars members of Praesepe

\begin{tabular}{|c|c|c|c|c|c|c|c|}
\hline $\mathrm{HD}$ or $\mathrm{BD}$ & $\begin{array}{l}\text { HR or } \\
\text { other }\end{array}$ & Sp & $m_{v}$ & $B_{2}-V_{1}$ & Variab. & $V \sin i$ & Remarks \\
\hline & Prae 23 & G & & 0.428 & $* * *$ & & \\
\hline $19^{\circ} 02050$ & Prae 34 & F2 V & 9.446 & 0.193 & & $<45$ & $\beta y$ \\
\hline 73174 & Prae 40 & $\mathrm{Am}$ & 7.759 & -0.010 & & 29 & вy \\
\hline $19^{\circ} 02052$ & Prae 47 & F4 V & 9.812 & 0.239 & ** & & By \\
\hline \multirow[t]{2}{*}{73345} & Prae 114 & F0 V & 8.152 & 0.000 & & 96 & вy \\
\hline & Prae 127 & G2 & 10.818 & 0.340 & $* * *$ & & By \\
\hline 73430 & Prae 143 & A9 V & 8.311 & 0.021 & ** & 73 & вy \\
\hline $20^{\circ} 02145$ & Prae 155 & F6 & 9.393 & 0.192 & ** & & By \\
\hline 73598 & Prae 212 & K0 III & 6.593 & 0.632 & $* * *$ & $<45$ & $\beta y$, DG \\
\hline 73616 & Prae 226 & F2 V & 8.899 & 0.111 & & 131 & $\beta y$ \\
\hline 73641 & Prae 227 & F2 V & 9.475 & 0.195 & * & 15 & вy \\
\hline 73617 & Prae 232 & F5 V & 9.221 & 0.156 & & 127 & By \\
\hline
\end{tabular}


Table 6 (Continued)

\begin{tabular}{|c|c|c|c|c|c|c|c|}
\hline $\mathrm{HD}$ or $\mathrm{BD}$ & $\begin{array}{l}\text { HR or } \\
\text { other }\end{array}$ & Sp & $m_{v}$ & $B_{2}-V_{1}$ & Variab. & $V \sin i$ & Remarks \\
\hline 73640 & Prae 239 & F4 V & 9.661 & 0.215 & & 32 & $\beta y$ \\
\hline $20^{\circ} 02157$ & Prae 250 & F6 V & 9.775 & 0.230 & & 120 & вy \\
\hline 73665 & $\begin{array}{l}3427 \\
\text { Prae } 253\end{array}$ & K0 III & 6.396 & 0.648 & $*$ & 0 & $4,6, \beta y, \mathrm{DG}$ \\
\hline $20^{\circ} 02161$ & Prae 271 & F2 V & 8.779 & 0.087 & $* * *$ & 86 & вy \\
\hline 73730 & $\begin{array}{l}\text { Prae } 286 \\
\text { Prae } 293\end{array}$ & Am & $\begin{array}{l}8.006 \\
9.836\end{array}$ & $\begin{array}{r}-0.011 \\
0.236\end{array}$ & $* *$ & 30 & $\begin{array}{l}\beta y \\
\beta y\end{array}$ \\
\hline $20^{\circ} 02170$ & Prae 295 & (F6) & 9.347 & 0.189 & & 95 & $\beta y$ \\
\hline 73746 & Prae 318 & Fo V & 8.644 & 0.079 & & 95 & вy \\
\hline 73763 & Prae 323 & A9 V & 7.814 & 0.018 & & 130 & By \\
\hline 73798 & Prae 340 & FO Vn & 8.467 & 0.062 & & 166 & вy \\
\hline 73819 & Prae 348 & A6 Vn & 6.766 & -0.023 & & 140 & $\beta y$ \\
\hline 73854 & Prae 370 & F5 V & 9.014 & 0.133 & & 116 & By \\
\hline $20^{\circ} 02180$ & Prae 396 & F4 V & 9.815 & 0.234 & & & вy \\
\hline 73937 & Prae 411 & F4 V & 9.321 & 0.170 & & 49 & $\beta y$ \\
\hline 73974 & Prae 428 & K0 III & 6.910 & 0.653 & & $<45$ & $\beta y$, DG \\
\hline 73993 & Prae 429 & FO V & & 0.093 & $* *$ & 195 & By \\
\hline 74028 & Prae 445 & A7 V & 7.962 & 0.001 & & 180 & $\beta y$ \\
\hline $20^{\circ} 02190$ & Prae 454 & - & 9.881 & 0.230 & $*$ & & $\beta y$ \\
\hline 74058 & Prae 459 & F2 V & 9.204 & 0.164 & & 130 & $\beta y$ \\
\hline $20^{\circ} 02192$ & Prae 472 & - & 9.765 & 0.213 & & & $\beta y$ \\
\hline $20^{\circ} 02193$ & Prae 478 & F4 V & 9.674 & 0.218 & & $<45$ & $\beta y$ \\
\hline 72779 & $\begin{array}{l}3387 \\
\text { PraeVL } 13\end{array}$ & G8 III & 6.584 & 0.429 & & 95 & $\beta y$ \\
\hline 72846 & $\begin{array}{l}\text { PraeVL } \\
166\end{array}$ & A5 V & 7.494 & -0.039 & & 140 & $\beta y$ \\
\hline
\end{tabular}

TABLE 7

Stars members of Coma Berenices

\begin{tabular}{llllrrrr}
\hline HD or BD & $\begin{array}{l}\text { HR or } \\
\text { other }\end{array}$ & Sp & $m_{v}$ & $B_{2}-V_{1}$ & Variab. & $V \sin i$ & Remarks \\
\hline 105805 & 4633 & A4 V & 6.014 & -0.055 & $* *$ & 172 & $2,7, \beta y$ \\
106103 & & F5 V & & 0.190 & $* * *$ & $<12$ & $\beta y$ \\
106691 & & F2 V & 8.090 & 0.188 & $* *$ & 30 & $\beta y$ \\
106946 & & F2 V & 7.836 & 0.158 & $* * *$ & 50 & $\beta y$ \\
107067 & & F8 V & 8.696 & 0.282 & & $\leqslant 12$ & $\beta y$ \\
107132 & & G0 V & 8.787 & 0.281 & & 12 & $\beta y$ \\
107131 & 4684 & A5 V & 6.423 & -0.022 & & 175 & $2,7, \beta y$ \\
107168 & 4685 & Am & 6.250 & -0.024 & & $\leqslant 12$ & $\beta y$ \\
107276 & & Am & 6.625 & -0.011 & & 95 & $\beta y$ \\
107399 & & G0 V & 9.016 & 0.326 & & & $\beta y$ \\
107611 & & (F7) & 8.503 & 0.237 & $*$ & 15 & $\beta y$ \\
107685 & & (F7) & 8.529 & 0.237 & $* *$ & $\leqslant 12$ & $\beta y$ \\
107877 & & F5 V & 8.358 & 0.219 & $* *$ & 20 & $\beta y$ \\
107966 & 4717 & A4 p & 5.163 & -0.085 & & 54 & $2,7, \beta y$ \\
108154 & & (F8) & 8.559 & 0.251 & & $\leqslant 12$ & \\
108226 & & F6 V & 8.337 & 0.221 & $* *$ & $\leqslant 12$ & $\beta y$ \\
108486 & & Am & 6.676 & -0.012 & $*$ & 30 & \\
109307 & 4780 & Am & & -0.066 & & 8 & 2,8 \\
\hline
\end{tabular}


TABLE 8.1

Stars with known spectral energy distribution, Willstrop

\begin{tabular}{rrllrrrl}
\hline HD or BD & $\begin{array}{l}\text { HR or } \\
\text { other }\end{array}$ & Sp & $m_{v}$ & $B_{2}-V_{1}$ & Variab. $V$ sin $i$ & Remarks \\
\hline & & & & & & & \\
1013 & 45 & M2 III & 4.837 & 1.188 & $* * *$ & & 1,8 \\
3196 & 142 & F8 V & & 0.315 & & 18 & DG \\
4628 & 222 & K2 V & & 0.549 & & & 3,8, DG \\
9270 & 437 & G8 III & 3.654 & 0.664 & & $<19$ & $\beta y$, DG \\
11636 & 553 & A5 V & 2.664 & -0.061 & $* *$ & 73 & $1,8, \beta y$ \\
14386 & 681 & (gMbe) & 4.684 & 1.500 & $* *$ & & \\
20630 & 996 & G5 V & & 0.407 & & $<17$ & $\beta y$, DG \\
23249 & 1136 & K0 IV & 3.548 & 0.601 & $* * *$ & $<17$ & $1,3,4,8$, DG \\
36673 & 1865 & F0 Ib & 2.607 & 0.018 & $* * *$ & 13 & $\beta y$ \\
129247 & 5478 & A3 III & 3.769 & -0.119 & & 156 & \\
142860 & 5933 & F6 V & 3.880 & 0.258 & $* *$ & 7 & $1,3,4,8, \beta y$ \\
166197 & 6788 & B1 V & 6.117 & -0.222 & & 14 & $\beta y$ \\
173667 & 7061 & F6 V & 4.194 & 0.249 & & $<, \beta y$ \\
203504 & 8173 & K1 III & 4.104 & 0.758 & & $<17$ & $\beta y$, DG \\
209747 & 8413 & K4 III & & 1.041 & $* *$ & $<17$ & DG \\
217987 & & M2 V & 7.356 & 1.131 & & & \\
& & & & & & & \\
\hline
\end{tabular}

All these stars have also to satisfy conditions 1 to 4 (except complementary information given in the column 'remarks'). For each star we give the colour indice $B_{2}-V_{1}$. The 7 heterochromatic colours normalised to $B$ can be found in (1971); the 7 monochromatic colours (deduced from the 7 heterochromatic colours) normalised to $G$ and the 7 effective wavelengths of the monochromatic colours can be obtained at the Geneva Observatory (Monochromatic colours Catalogue). Let us point out that the $\lambda_{\text {eff }}$ of filter $U$ may not always be very accurate for stars having a large Balmer discontinuity.

Many stars are common to several tables; the numbers of the tables are given in the 'remarks' column. The magnitudes given are the $V$ magnitudes established by Rufener and Maeder (1972), the magnitudes in brackets are taken from Literature. The spectral classifications given in tables 3 to 7 are taken from the catalogues of Jaschek $e t$ al. (1964) and from its extension by Kennedy. In the remarks column we also indicate by $\beta y$ the stars also measured in the $u v b y \beta$ system, the general catalogue of which is being prepared by Lindemann and Hauck (1973); and by DG the cool stars measured in the Copenhagen system (1970).

The selection presented here gathers together stars which may serve to calibrate criteria for $M_{v}, \mathrm{Sp},[\mathrm{Fe} / \mathrm{H}]$, etc. But these are also stars to which we believe it is important to give some attention and to attempt to determine the fundamental parameters $M_{v}, \theta_{\text {eff }}, \log g, \chi$. The proposed selection should be enriched with hot stars of well known absolute magnitude and reddening. For this purpose, one must measure young clusters of well determined distance modulus. 
TABLE 8.2

Stars with known spectralenergy distribution, Terechtchenko and Kharitonov

\begin{tabular}{|c|c|c|c|c|c|c|c|}
\hline $\mathrm{HD}$ or $\mathrm{BD}$ & $\begin{array}{l}\text { HR or } \\
\text { other }\end{array}$ & $\mathrm{Sp}$ & $m_{v}$ & $B_{2}-V_{1}$ & Variab. & $V \sin i$ & Remarks \\
\hline 3196 & 142 & F8 V & & 0.315 & & 18 & DG \\
\hline 4727 & 226 & B5 V & 4.525 & -0.242 & & 75 & By \\
\hline 8538 & 403 & A5 V & 2.653 & -0.045 & ** & 116 & $1,8, \beta y$ \\
\hline 11636 & 553 & A5 V & 2.664 & -0.061 & & 73 & $1,8, \beta y$ \\
\hline 16970 & 804 & A2 V & & -0.087 & & 183 & \\
\hline 20320 & 984 & Am & 4.797 & 0.023 & * & 68 & $\beta y$ \\
\hline 22928 & 1122 & B5 III & 3.012 & -0.240 & & 271 & $1,8, \beta y$ \\
\hline 25490 & 1251 & A1 V & & -0.127 & & 71 & $2,8, \beta y$ \\
\hline 35468 & 1790 & B2 III & 1.634 & -0.297 & & 64 & $1,8, \beta y$ \\
\hline 58715 & 2845 & B8 V & & -0.208 & & 270 & $\beta y$ \\
\hline 71155 & 3314 & $\mathrm{~A} 0 \mathrm{~V}$ & 3.902 & -0.159 & & 122 & $1,2,8, \beta y$ \\
\hline 77327 & 3594 & B9n & 3.584 & -0.147 & & 219 & $2,8, \beta y$ \\
\hline 79469 & 3665 & B9.5 V & - & -0.208 & & 86 & $2,8, \beta y$ \\
\hline 87901 & 3982 & B7 V & 1.375 & -0.219 & & 354 & $1,8, \beta y$ \\
\hline 90089 & 4084 & F5 IV & 5.259 & 0.180 & * & 107 & $\beta y$ \\
\hline 97633 & 4359 & A2 V & 3.320 & -0.156 & & 14 & $1,2,8, \beta y$ \\
\hline 102870 & 4540 & F8 V & 3.636 & 0.301 & & 0 & $1,3,4,8, \beta y$ \\
\hline 103287 & 4554 & A0 V & 2.452 & -0.154 & & 163 & $1,2,8, \beta y$ \\
\hline 106112 & 4646 & Am & 5.145 & 0.109 & & 69 & By \\
\hline 108382 & 4738 & A2 & 4.986 & -0.074 & & 89 & 2,8 \\
\hline 110379 & 4825 & FO V & 2.766 & 0.141 & $*$ & 27 & $1,3,8, \beta y$ \\
\hline 120315 & 5191 & B3 V & 1.856 & -0.258 & & 216 & By \\
\hline 123299 & 5291 & A0 III & 3.659 & -0.180 & $* * *$ & 12 & 1,8 \\
\hline 129247 & 5478 & A3 III & 3.769 & -0.119 & & 156 & \\
\hline 135742 & 5685 & B8 V & & -0.209 & * & 230 & $1,8, \beta y$ \\
\hline 148112 & 6117 & Alp & & -0.156 & & 28 & \\
\hline 170153 & 6927 & F7 V & 3.546 & 0.279 & ** & 11 & $4,8, \beta y$ \\
\hline 202444 & 8130 & F0 IV & & 0.185 & & 94 & \\
\hline 207098 & 8322 & Am & & 0.095 & * & 104 & By \\
\hline 212061 & 8518 & B9 III & & -0.193 & & 82 & By \\
\hline 218658 & 8819 & G2 III & 4.401 & 0.510 & & 22 & DG \\
\hline 224617 & 9072 & F4 IV & & 0.206 & $*$ & 34 & $\beta y$ \\
\hline
\end{tabular}

\section{Stars With Known Spectral Energy Distribution}

In Table 8 we give the 7 colours of the stars whose spectral energy distribution is given by Willstrop (1965) and by Terechtchenko and Kharitonov (1972). The selected stars do not satisfy the 4 conditions given in paragraph 3 . They have a weight $p \geqslant 3$ in the $U V B B_{1} B_{2} V_{1} G$ photometry, but can be binaries, reddened, and have a high rotational velocity. Nevertheless, they are useful for the photometrist who can use them to check the quality of the determination of his system's bandpasses.

\section{References}

Cayrel, R. and Cayrel de Strobel, G.: 1966, 'Abundance Determinations from Stellar Spectra', in Ann. Rev. Astron. Astrophys. 4. 
Cowley, A. Cowley, Ch., Jaschek, M., and Jaschek, C.: 1969, Astron. J. 74, 375.

Dickow, R., Gyldenkerne, K., Hansen, L., Jacobsen, P. U., Kjaergaard, K., and Olsen, E. H.: 1970, Astron. Astrophys. Suppl. Ser. $2,1$.

Gliese, W.: 1969, 'Catalogue of Nearby Stars', Veröffentl. Astron. Rechen-Inst. Heidelberg No. 22.

Golay, M.: 1972, Vistas in Astronomy 14, in press.

Golay, M.: 1969, in O. Gingerich (ed.), Theory and Observations of Normal Stellar Atmospheres, M.I.T. Press, Cambridge.

Golay, M.: 1971, in Ch. Fehrenbach and B. E. Westerlund (eds.), 'Spectral Classification and Multicolour Photometry', IAU Symp. 50, 145.

Jaschek, C., Condé, H., and Sierra, A.: 1969, Catalogue of Stellar Spectra Classified, in the MorganKeenan System, La Plata.

Johnson, H. L. and Morgan, W. W.: 1953, Astrophys. J. 117, 313.

Kennedy, P. M.: 1970, Extension du Catalogue de Jaschek et al., not published.

Lindemann, E. and Hauck, B.: 1973, Astron. Astrophys., Suppl. 11, 119.

Maeder, A. and Peytremann, E.: 1970, Astron. Astrophys. 7, 120.

Maeder, A. and Peytremann, E.: 1972, Astron. Astrophys. 21, 279.

Morgan, W. W. and Roman, N. G.: 1950, Astrophys. J. 112, 362.

Morgan, W. W., Harris, D. L., and Johnson, H. L.: 1953, Astrophys. J. 118, 92.

Peytremann, E.: 1970, Thesis, Obs. de Genève.

Powell, A. L. T.: 1970, Monthly Notices Roy. Astron. Soc. 148, 477.

Rufener, F. G.: 1971, Astron. Astrophys. Suppl. Ser. 3. 181.

Rufener, F. and Maeder, A.: 1972, this volume, p. 156.

Terechtchenko, V. M. and Kharitonov, A. V.: 1972, Trudy Astrofiz. Inst. Alma-Ata, 21.

Willstrop, R. V.: 1965, Men. Roy. Astron. Soc. 69, part 3.

\section{DISCUSSION}

Pecker: When trying to apply to observed stars the very fine rotating models built by Maeder, for example, we should keep in mind that the measured ' $V \sin i$ ' plotted in the tables of Golay are not necessary measured rotations, they are just measurements of broadening, and stellar lines can be broadened by other causes, such as macro-velocity fields. Therefore, the 'rotating models' are not necessarily adequate to fit the observations.

Kodaira: I am of the same opinion as Dr Pecker, but as the zeroth approximation, one can regard the broadening of lines as the result of the rotation. It is worthwhile to try to establish the possible rotation effect observationally. But Dr Golay limits the value of $V \sin i$ to below $150 \mathrm{~km} \mathrm{~s}^{-1} \mathrm{~s}$, in order to avoid the influence of the stellar rotation. In doing so, however, you would bias your data, by mixing the intrinsic slow rotators with stars of high or moderate rotational velocity whose axises have small or moderate inclination (near 'pole-on'). My suggestion is to extend the standard stars as far as possible to a higher value of $V \sin i(\sim 350)$, so far as no emission lines are observed.

Maeder: In connexion with Prof. Pecker's remark, I will firstly add that the fact that we do not know the law of rotation inside the stars is a black point in the theory of rotating models. In that respect, we may perhaps hope that the comparisons between observations and models bring some information on this subject. Secondly, so-called observable quantities like $V \sin i$ in fact are estimated by means of theoretical models.

Garrison: This may seem to be a comical remark, but I will make it semi-seriously. Perhaps we should choose stars with large $V \sin i$ as standards. At least then we know they are not pole-on and it should be a more homogeneous group.

FitzGerald: To add to Dr Garrison's remark that perhaps the fast rotation should be used as standards, it should be remarked that there was a paper at Athens suggesting that Am stars were slow rotators, in which element differentiation could occur, whereas in the moderate rotation mixing occurs preventing element differentiation. Thus the 'normal' stars suitable for standards should be the moderate rotators.

Hanbury-Brown: The angular diameters of 32 stars have been measured at Narrabri Observatory ranging in spectral type from O5 to F8. I will send a list of these stars to Prof. Golay for consideration as possible members of his list of standard stars.

Pecker: When one shifts from a group of stars of a given brightness to a less bright, methods of 
observation are changed. For example, (1) very bright stars are studied with very large dispersion; abundances etc. are determined. (2) bright stars are studied with lower spectrographic resolution and are more numerous. (3) still more numerous, less bright stars are available for 7-colour photometry (for example) etc.... The stars to be used as standards for group $(n)$ have to be taken in groups (1) (2)... $(n-1)$, but the smaller the $n$ is, the smaller is the number of standard stars that are not monsters, that are 'safe'. By going to larger and larger groups, one improves the sampling, one deteriorates the quality of the 'standard' physical characteristics of this sampling. The whole thing is essentially a matter of successive approximations, and of the choice of 'proper equilibrium' between the completeness of the sampling and the physical meaning of this sampling.

Lamla: Which effects have faint lines in your 7-colour system? You compare your integrated brightness values with model intensities which give, I guess, the continuum without lines.

Golay: No, we use the models of Peytremann (Thesis, Geneva (1970) which include the opacities of metallic lines.

Garrison: It is perhaps useful to clarify the situation regarding 'standard' stars in the MK system. For this purpose we can talk about fundamental standards, which are those stars to which the MK system is anchored; about primary standards, which are those stars that have been studied extremely carefully, such as the ones listed in the Johnson-Morgan paper; about secondary standards, which are stars within a given set of plates which fit the above standards and look very normal; and about the non-standard types which are stars that are observed carefully and classified carefully using the MK system. These latter stars should not be used with the same weight, in general, as the fundamental or primary standards as representatives of the MK system. In Prof. Golay's paper, for example, he has referred to the paper by the Cowley's and the Jaschek's as standard. I think that Prof. Jaschek would agree that his paper is in the last category.

Hack: Because of the uncertainty of oscillator strengths and because of the difficulty to fit stellar atmospherical models and observations, probably the safest way to derive atmospherical chemical composition is the method of the differential curve of growth. Hence we need standard stars for which we have good data on spectral energy distribution, Balmer discontinuity etc. so as to have reliable data on $T_{\text {eff }}$ and $g$, and $V \sin i<20 \mathrm{~km} \mathrm{~s}^{-1}$, and to correlate all these standards to the Sun by a 'grid' of standards covering the whole HR diagram, that is by comparing a G0 V to the Sun, a F8 V to the GOV and so on.

Keenan: In connection with your list of standard stars for observation in as many ways as possible I would recommend first the inclusion of all the stars in the lists that Morgan and I will include in our review article that is coming out in Annual Reviews of Astronomy and Astrophysics next year. These lists are not ready yet, but in the meantime I shall send you soon a short list of some stars, mostly fainter than $V=6.5$, that can be considered as type stars of some of the special population groups. If you do observe these in your colour system it will be very interesting to see how you distinguish them from the ordinary stars of types $\mathbf{G}, \mathbf{K}$ and $M$. The types that I give in the list represent my most recent ones.

Murray: I would like to put in a plea for more late type dwarfs among the standards; there is only one in Golay's lists. There are obvious difficulties due to faintness and probable duplicity of many of these stars, but accurate calibration of their luminosities are very important. Fortunately many of these stars have good trigonometric parallaxes, and soon there will be more parallaxes available for Vyssotsky's stars which are on the observing lists of several parallax observers.

Gliese: The distance determinations of Vyssotsky's stars (which are dK8 to dM2) is not sufficient since the maximum of the luminosity function in the solar neighbourhood is supposed to be in the region of the $\mathrm{dM} 8$ stars. Probably the most promising way is by the parallax program of the USNO in combination with $R, I$ measurements which allow the calibration of the $\left(M_{v}, R-I\right)$ relation of these very red dwarfs. This relation should be used for late-type red dwarfs, for instance, near the galactic poles.

Jones: I have observed $700 \mathrm{M}$ stars with $30 \AA \AA$ interference filters:

7460 Window

$7100 \mathrm{TiO}$

$6830 \mathrm{Ca} \mathrm{H}$

6076 Window.

As was first shown by Ohman $\mathrm{Ca} \mathrm{H}$ is luminosity sensitive. On the present system $\mathrm{TiO}$ : $\mathrm{CaH}$ is a dwarf/giant discriminator with amplitude $\sim 0.4 \mathrm{mag}$. 7460-6076 correlates well with $R-I$ and photometric parallaxes can be determined from the $m_{7460} \mathrm{vs} m_{7460}-m_{6076}$ plot. The TiO strength correlates 
well with that observed by Wing. At the Cordoba Symposium he proposed a new spectral type scale for dwarfs, based on giants with the same TiO strength. I suggest the same standards be adopted here. In particular I confirm Wing's conclusion that Proxima Centauri is $\sim .04$ of a spectral type earlier than Wolf 359. I have observed the $100 \mathrm{M}$ stars discovered by McCarthy and his co-workers in the South Galactic Cap. About 25 are dwarfs with median proper motion $\sim 0$ "!2. Only a couple are closer than 20 pars. Of course, the volume of space searched at M6 V is much smaller than at M0 V.

Pecker: Theoreticians want to get from obsel vers $L, M, R,-$ to induce results on evolutionary properties, etc. It seems to me that from our point of view, interesting stars are necessarily falling in the region of the conceptual ( $M_{v}$, apparent Radius, apparent binary separation) diagram where data at present are sufficiently well known. 'Standards' are there in small numbers; but the use of photometry, using this small number of objects as primary standards, may extend the number of stars that could be used as secondary standards.

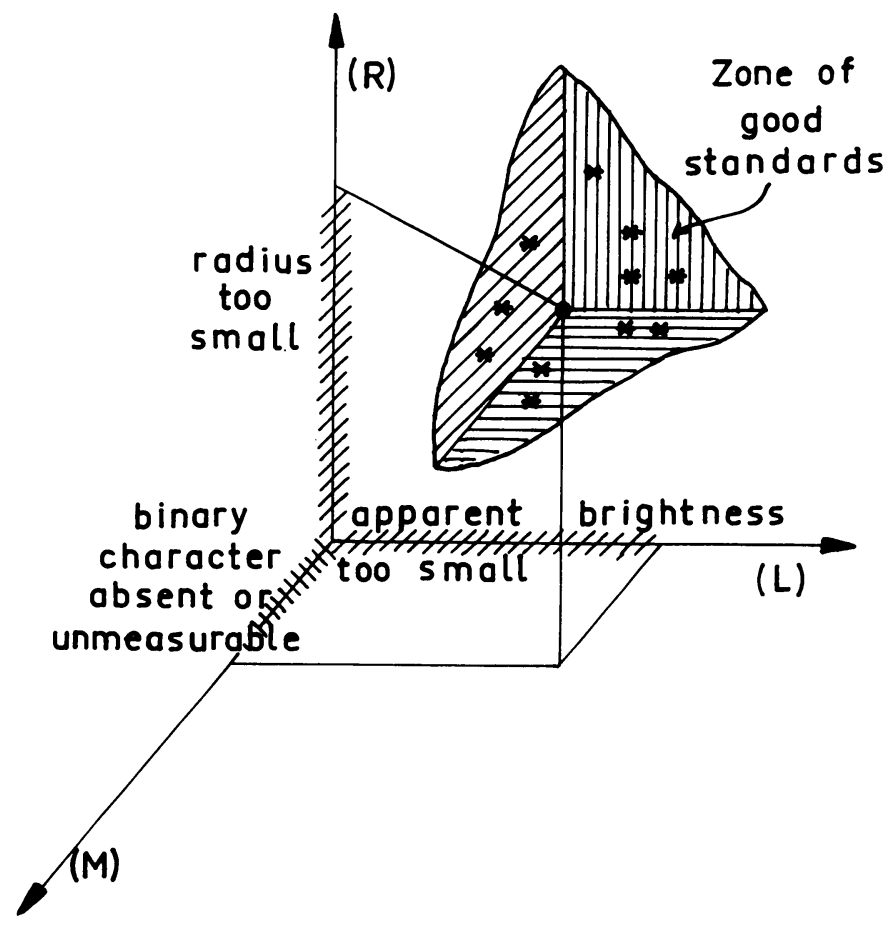

Fig. 6 